\title{
EXISTENCE OF POSITIVE SOLUTIONS FOR A CLASS OF SINGULAR AND QUASILINEAR ELLIPTIC PROBLEMS WITH CRITICAL EXPONENTIAL GROWTH
}

\author{
Suellen Cristina Q. Arruda, Giovany M. Figueiredo \\ and Rubia G. Nascimento
}

\author{
Universidade Federal do Pará - UFPA, Faculdade de Ciências Exatas e Tecnologia \\ CEP: 68440-000, Abaetetuba-PA, Brazil; scqarruda@ufpa.br \\ Universidade de Brasília - UNB, Departamento de Matemática \\ CEP: 70910-900, Brasília-DF, Brazil; giovany@unb.br \\ Universidade Federal do Pará - UFPA, Instituto de Ciências Exatas e Naturais \\ CEP: 66075-110, Belém-PA, Brazil; rubia@ufpa.br
}

\begin{abstract}
In this paper we use Galerkin method to investigate the existence of positive solution for a class of singular and quasilinear elliptic problems given by

$$
\begin{cases}-\operatorname{div}\left(a_{0}\left(|\nabla u|^{p_{0}}\right)|\nabla u|^{p_{0}-2} \nabla u\right)=\frac{\lambda_{0}}{u^{\beta_{0}}}+f_{0}(u), u>0 & \text { in } \Omega \\ u=0 & \text { on } \partial \Omega\end{cases}
$$

and its version for systems given by

$$
\begin{cases}-\operatorname{div}\left(a_{1}\left(|\nabla u|^{p_{1}}\right)|\nabla u|^{p_{1}-2} \nabla u\right)=\frac{\lambda_{1}}{u^{\beta_{1}}}+f_{1}(v) & \text { in } \Omega \\ -\operatorname{div}\left(a_{2}\left(|\nabla v|^{p_{2}}\right)|\nabla v|^{p_{2}-2} \nabla v\right)=\frac{\lambda_{2}}{v^{\beta_{2}}}+f_{2}(u) & \text { in } \Omega, \\ u, v>0 & \text { in } \Omega, \\ u=v=0 & \text { on } \partial \Omega\end{cases}
$$

where $\Omega \subset \mathbf{R}^{N}$ is bounded smooth domain with $N \geq 3$ and for $i=0,1,2$ we have $2 \leq p_{i}<N, 0<$ $\beta_{i} \leq 1, \lambda_{i}>0$ and $f_{i}$ are continuous functions. The hypotheses on the $C^{1}$-functions $a_{i}: \mathbf{R}^{+} \rightarrow \mathbf{R}^{+}$ allow to consider a large class of quasilinear operators.
\end{abstract}

\section{Introduction}

In a celebrated paper in 1976 [39], Stuart considered the problem

$$
L(u)=f(x, u) \text { in } \Omega, u=\phi(x) \text { on } \partial \Omega,
$$

where $\Omega$ is a bounded domain in $\mathbf{R}^{N}, N \geq 2, L$ be a second order linear elliptic operator and $f(x, p) \rightarrow \infty$ as $p \rightarrow 0$. Problems of this type are called singular and arise in the theory of heat conduction in electrically conducting materials. Moreover, they have wide application to physical models such as non-Newtonian fluids, boundary layer phenomena for viscous fluids, chemical heterogenous, see [7] and [8]. Using the maximum principle Stuart establishes the existence of non-negative solutions of this problem and constructs iteration schemes that converge to a solution.

In 1997, Crandall, Rabinowitz, Tartar [14] go back to study this class of problems, where $L$ is assumed to be a linear second order elliptic operator that satisfies a maximum principle. In the first part the existence of a classical solution continuous

https://doi.org/10.5186/aasfm.2021.4626

2020 Mathematics Subject Classification: Primary 35J20, 35J50; Secondary 58E05.

Key words: Galerkin method, Exponential growth, Trudinger-Moser inequality, HardySobolev inequality. 
up to the boundary is proved by means of sub-supersolutions method. The second part of the paper is devoted to a detailed study of the continuity properties of a solution for special nonlinearities independent of $x$.

More recently, the version with $L=-\Delta$ and $f(x, u)=\frac{1}{u^{\alpha}}+\lambda|\nabla u|^{p}+\sigma$, where $\alpha>$ $0, \sigma \geq 0,0<p \leq 2, \phi=0$ was studied in [19] and [43]. The gradient in this equation is called convection term. The version without the convection term was studied in [37]. Another important results can be found in [4, 5, 9, 10, 13, 15, 17, 22, 28, 32]. The system versions were studied in $[1,12,24,30,42]$.

In [20] Giacomoni, Prashanth and Sreenadh studied a problem with $N$-Laplacian such that the nonlinearity grows like $\exp \left(|t|^{N / N-1}\right)$ at infinity and like $\frac{1}{t^{\alpha}}$ at the origin. A similar problem with the Laplacian operator in $\mathbf{R}^{2}$ was studied by Saoudi and Kratou in [35]. In [16] Dhanya, Prashanth, Sreenadh and Tiwari considered the singular case with critical exponential growth and discontinous nonlinearity. The inhomogeneous singular Neumann case was studied in [36]. The multiplicity results was considered in [33]. The version in $\mathbf{R}^{N}$ with $N$-Laplacian and critical exponential growth was studied in [2].

We are going back to our problem in order to enunciate the hypotheses on the functions $a_{i}$ and $f_{i}$. More precisely, the hypotheses on functions $a_{i}: \mathbf{R}^{+} \longrightarrow \mathbf{R}^{+}$of $C^{1}$ class are the following:

$\left(a_{1}\right)$ There exist constants $k_{1}, k_{3}, k_{4} \geq 0$ and $k_{2}>0$, such that

$$
k_{1} t^{p_{i}}+k_{2} t^{N} \leq a_{i}\left(t^{p_{i}}\right) t^{p_{i}} \leq k_{3} t^{p_{i}}+k_{4} t^{N}, \quad \text { for all } t \geq 0
$$

$\left(a_{2}\right)$ The function

$$
t \longmapsto a_{i}\left(t^{p_{i}}\right) t^{p_{i}-2} \text { is increasing. }
$$

The functions $f_{i}: \mathbf{R} \longrightarrow \mathbf{R}$ are continuous satisfying the following properties:

$\left(f_{1}\right)$ There exists $\alpha_{0}>0$ such that the exponential growth conditions at infinity are given by:

$\lim _{t \rightarrow \infty} \frac{f_{i}(t)}{\exp \left(\alpha|t|^{\frac{N}{N-1}}\right)}=0$ for $\alpha>\alpha_{0}$ and $\lim _{t \rightarrow \infty} \frac{f_{i}(t)}{\exp \left(\alpha|t|^{\frac{N}{N-1}}\right)}=\infty$ for $0<\alpha<\alpha_{0}$.

$\left(f_{2}\right)$ The growth condition at the origin:

$$
\lim _{t \rightarrow 0^{+}} \frac{f_{i}(t)}{t^{p_{i}-1}}=0 .
$$

$\left(f_{3}\right)$ There exists $\gamma_{i}>N$ such that

$$
f_{i}(t) \geq t^{\gamma_{i}-1}, \text { for all } t \geq 0 \text {. }
$$

Since we are looking for positive solution, in this paper we consider $f_{i}(t)=0$ for all $t<0$.

The purpose in the first part of this article is to prove the existence of solution for the following class of singular problems

$$
\begin{cases}-\operatorname{div}\left(a_{0}\left(|\nabla u|^{p_{0}}\right)|\nabla u|^{p_{0}-2} \nabla u\right)=\frac{\lambda_{0}}{u^{\beta_{0}}}+f_{0}(u) & \text { in } \Omega, \\ u>0 & \text { in } \Omega, \\ u=0 & \text { on } \partial \Omega,\end{cases}
$$

where $\Omega \subset \mathbf{R}^{N}$ is a bounded smooth domain, $N \geq 3,2 \leq p_{0}<N$ and $0<\beta_{0} \leq 1$, $\lambda_{0}$ are real parameters.

The main result in the first part is: 
Theorem 1.1. Assume that conditions $\left(a_{1}\right)-\left(a_{2}\right)$ and $\left(f_{1}\right)-\left(f_{3}\right)$ hold. Then, there exists $\lambda^{*}>0$ such that the problem (1.1) has a positive weak solution for every $\lambda_{0} \in\left(0, \lambda^{*}\right)$.

In the second part of this article we study the following system

$$
\begin{cases}-\operatorname{div}\left(a_{1}\left(|\nabla u|^{p_{1}}\right)|\nabla u|^{p_{1}-2} \nabla u\right)=\frac{\lambda_{1}}{u^{\beta_{1}}}+f_{1}(v) & \text { in } \Omega, \\ -\operatorname{div}\left(a_{2}\left(|\nabla v|^{p_{2}}\right)|\nabla v|^{p_{2}-2} \nabla v\right)=\frac{\lambda_{2}}{v^{\beta_{2}}}+f_{2}(u) & \text { in } \Omega, \\ u, v>0 & \text { in } \Omega, \\ u=v=0 & \text { on } \partial \Omega,\end{cases}
$$

where $\Omega \subset \mathbf{R}^{N}$ is bounded smooth domain with $N \geq 3$ and for $i=1,2$ we have $2 \leq p_{i}<N, 0<\beta_{i} \leq 1, \lambda_{i}>0, a_{i}: \mathbf{R}^{+} \rightarrow \mathbf{R}^{+}$are functions of $C^{1}$ class and $f_{i}: \mathbf{R} \rightarrow \mathbf{R}$ are continuous functions with exponential growth.

The main result of this second part is the following:

Theorem 1.2. Assume that, for $i=1,2, a_{i}$ satisfy $\left(a_{1}\right)-\left(a_{2}\right)$ and $f_{i}$ satisfy $\left(f_{1}\right)-$ $\left(f_{3}\right)$. Then, there exists $\lambda^{*}>0$ such that the problem (1.2) has a positive weak solution for every $0<\lambda_{1}+\lambda_{2}<\lambda^{*}$.

We will give some examples of functions $a_{i}$ in order to illustrate the degree of generality of the kind of problems studied here.

Example 1.1. Considering $a_{i}(t)=t^{\frac{N-p_{i}}{p_{i}}}$, we have that the function $a_{i}$ satisfies the hypotheses $\left(a_{1}\right)-\left(a_{2}\right)$ with $k_{1}=k_{3}=0$ and $k_{2}=k_{4}=1$. Hence, Theorems 1.1 and 1.2 are valid for the operator $-\Delta_{N} u$.

Example 1.2. Considering $a_{i}(t)=1+t^{\frac{N-p_{i}}{p_{i}}}$, we have that the function $a_{i}$ satisfies the hypotheses $\left(a_{1}\right)-\left(a_{2}\right)$ with $k_{1}=k_{2}=k_{3}=k_{4}=1$. Hence, Theorems 1.1 and 1.2 are valid for the operator $-\Delta_{p_{i}} u-\Delta_{N} u$.

Problems with this operator come from a general reaction-diffusion system:

$$
u_{t}=\operatorname{div}[D(u) \nabla u]+c(x, u),
$$

where $D(u)=\left(|\nabla u|^{p-2}+|\nabla u|^{N-2}\right)$. This system has a wide range of applications in physics and related sciences, such as biophysics, plasma physics and chemical reaction design. In such applications, the function $u$ describes a concentration, the first term on the right-hand side of (1.3) corresponds to the diffusion with a diffusion coefficient $D(u)$; whereas the second one is the reaction and relates to source and loss processes. Typically, in chemical and biological applications, the reaction term $c(x, u)$ is a polynomial of $u$ with variable coefficients (see $[11,23,29,41]$ ).

Beneath we present some other examples that are also interesting from mathematical point of view.

Example 1.3. Considering $a(t)=1+\frac{1}{(1+t)^{\frac{p_{i}-2}{p_{i}}}}$, we have that the function $a$ satisfies the hypotheses $\left(a_{1}\right)-\left(a_{2}\right)$ with $k_{1}=1, k_{3}=2, k_{4}=0$ and $k_{2}>0$. Hence, Theorems 1.1 and 1.2 are valid for the operator

$$
-\operatorname{div}\left(|\nabla u|^{p_{i}-2} \nabla u+\frac{|\nabla u|^{p_{i}-2} \nabla u}{\left(1+|\nabla u|^{p_{i}}\right)^{\frac{p_{i}-2}{p_{i}}}}\right) .
$$


Example 1.4. Considering $a_{i}(t)=1+t^{\frac{N-p}{p}}+\frac{1}{(1+t)^{\frac{p_{i}-2}{p_{i}}}}$, it follows that the function $a$ satisfies the hypotheses $\left(a_{1}\right)-\left(a_{2}\right)$ with $k_{1}=k_{2}=k_{4}=1$ and $k_{3}=2$. Hence, Theorems 1.1 and 1.2 are valid for the operator

$$
-\Delta_{p_{i}} u-\Delta_{N} u-\operatorname{div}\left(\frac{|\nabla u|^{p_{i}-2} \nabla u}{\left(1+|\nabla u|^{p_{i}}\right)^{\frac{p_{i}-2}{p_{i}}}}\right) .
$$

Other combinations can be made with the functions presented in the examples above, generating very interesting elliptic problems from the mathematical point of view.

Below we list what we believe that are the main contributions of our paper:

1) In [13] and [12] were studied a singular problem and a singular system, respectively, with this general operator. But the nonlinearities have polynomial growth.

2) In [16], [20], [35] and [36] were studied the singular case with a nonlinearities with exponential growth. However, here we study problems with a general operator which brings some technical difficulties.

3) Here we use Galerkin method that was not used in the papers above cited.

The plan of the paper is the following: In Section 2 we recall some preliminary results for the scalar case. In Section 3 we study an auxiliary problem for the scalar case. We show existence of solution of the auxiliary problem in Section 4 . In Section 5 we prove Theorem 1.1. In Section 6 we study an auxiliary problem for the system case. We show existence of solution of the auxiliary problem in Section 7. In Section 8 we prove Theorem 1.2

\section{Preliminary results for the scalar case}

Let us consider the Sobolev space $W_{0}^{1, N}(\Omega)$ endowed with the norm

$$
\|u\|_{1, N}=\left(\int_{\Omega}|\nabla u|^{N} d x\right)^{\frac{1}{N}} .
$$

We say that $u \in W_{0}^{1, N}(\Omega)$ is a weak solution of the problem (1.1) if $u>0$ in $\Omega$ and it verifies

$$
\int_{\Omega} a_{0}\left(|\nabla u|^{p_{0}}\right)|\nabla u|^{p_{0}-2} \nabla u \nabla \phi d x-\lambda_{0} \int_{\Omega} \frac{\phi}{u^{\beta_{0}}} d x-\int_{\Omega} f_{0}(u) \phi d x=0,
$$

for all $\phi \in W_{0}^{1, N}(\Omega)$. In this paper, we work with operator $T_{i}: W_{0}^{1, N}(\Omega) \rightarrow\left(W_{0}^{1, N}(\Omega)\right)^{\prime}$ such that

$$
\left\langle T_{i} u_{i}, \phi_{i}\right\rangle=\int_{\Omega} a_{i}\left(\left|\nabla u_{i}\right|^{p_{i}}\right)\left|\nabla u_{i}\right|^{p_{i}-2} \nabla u_{i} \nabla \phi_{i} d x .
$$

A straightforward calculation shows that $T_{i}$ is continuous. Furthermore, $T_{i}$ is monotone and coercive, see [12, Lemma 1].

Firstly, we recall some important results due to Trudinger-Moser [31, 40] and Hardy-Sobolev [25]. A version of Trudinger-Moser inequality for systems can be found in [3].

Theorem 2.1. (Trudinger-Moser inequality) For every $u \in W_{0}^{1, N}(\Omega)$ and $\alpha>0$, then

$$
\exp \left(\alpha u^{\frac{N}{N-1}}\right) \in L^{1}(\Omega)
$$


and there exists a constant $M>0$ such that

$$
\sup _{\|u\|_{1, N} \leq 1} \int_{\Omega} \exp \left(\alpha u^{\frac{N}{N-1}}\right) d x \leq M
$$

for every $\alpha \leq \alpha_{N}:=N w_{N-1}^{\frac{1}{N-1}}$, where $w_{N-1}$ is the $(N-1)$-dimensional measure of $(N-1)$ sphere.

Theorem 2.2. (Hardy-Sobolev inequality) If $u \in C^{1}(\bar{\Omega}) \cap W_{0}^{1, p}(\Omega)$ with $1<$ $p \leq N$, then $\frac{u}{C d^{\tau}} \in L^{r}(\Omega)$, for $\frac{1}{r}=\frac{1}{p}-\frac{1-\tau}{N}, 0<\tau \leq 1$ and

$$
\left|\frac{u}{C d^{\tau}}\right|_{L^{r}(\Omega)} \leq|\nabla u|_{L^{p}(\Omega)},
$$

where $d(x)=\operatorname{dist}(x, \partial \Omega)$ and $C$ is a positive constant which does not depend on $x$.

Our approach in the study of problem (1.1) and system (1.2) rests heavily on the following Weak Comparison Principle proved in [12, Lemma 2].

Lemma 2.1. If $\Omega$ is a bounded domain and if $u_{i}, v_{i} \in W_{0}^{1, N}(\Omega)$ satisfy

$$
\left\{\begin{array}{l}
-\operatorname{div}\left(a_{i}\left(\left|\nabla u_{i}\right|^{p_{i}}\right)\left|\nabla u_{i}\right|^{p_{i}-2} \nabla u_{i}\right) \leq-\operatorname{div}\left(a_{i}\left(\left|\nabla v_{i}\right|^{p_{i}}\right)\left|\nabla v_{i}\right|^{p_{i}-2} \nabla v_{i}\right) \text { in } \Omega, \\
u_{i} \leq v_{i} \text { on } \partial \Omega
\end{array}\right.
$$

then $u_{i} \leq v_{i}$ a.e. in $\Omega$.

We observe that, from $\left(f_{1}\right)-\left(f_{2}\right)$, for all $\delta>0$ and for all $\alpha>\alpha_{0}$, there exists $C_{\delta}>0$ such that

$$
\left|f_{i}(t) t\right| \leq \delta|t|^{p_{i}}+C_{\delta}|t|^{q_{i}} \exp \left(\alpha|t|^{\frac{N}{N-1}}\right),
$$

for all $q_{i} \geq 0$. In this paper, we will use $q_{i}>N$.

\section{Auxiliary problem for the scalar case}

For each $\varepsilon>0$, we consider the following auxiliary problem

$$
\begin{cases}-\operatorname{div}\left(a_{0}\left(|\nabla u|^{p_{0}}\right)|\nabla u|^{p_{0}-2} \nabla u\right)=\frac{\lambda_{0}}{(u+\varepsilon)^{\beta_{0}}}+f_{0}(u) & \text { in } \Omega, \\ u>0 & \text { in } \Omega, \\ u=0 & \text { on } \partial \Omega,\end{cases}
$$

where the functions $a_{0}$ and $f_{0}$ satisfy the hypotheses of the Theorem 1.1.

To prove Theorem 1.1, we first show the existence of a solution for the problem (3.1). For this, we will use the Galerkin method together with the following fixed point theorem, see [38] and [27, Theorem 5.2.5].

Lemma 3.1. Let $G: \mathbf{R}^{d} \longrightarrow \mathbf{R}^{d}$ be a continuous function such that $\langle G(\xi), \xi\rangle \geq 0$ for every $\xi \in \mathbf{R}^{d}$ with $|\xi|=r$ for some $r>0$. Then, there exists $z_{0} \in \bar{B}_{r}(0)$ such that $G\left(z_{0}\right)=0$.

The main result in this section is the following:

Lemma 3.2. For each $0<\varepsilon<1$, there exists $\lambda^{*}>0$ such that the problem (3.1) has a positive weak solution for every $\lambda_{0} \in\left(0, \lambda^{*}\right)$.

Proof. Let $B=\left\{e_{1}, e_{2}, \ldots, e_{m}, \ldots\right\}$ be a Schauder basis of $W_{0}^{1, N}(\Omega)$. For each $m \in \mathbf{N}$, define

$$
W_{m}=\left[e_{1}, e_{2}, \ldots, e_{m}\right]
$$


to be the finite-dimensional space generated by $\left\{e_{1}, e_{2}, \ldots, e_{m}\right\}$. Note that the spaces $\left(W_{m},\|\cdot\|_{m}\right)$ and $\left(\mathbf{R}^{m},|\cdot|_{s}\right)$ are isometrically isomorphic by natural mapping

$$
S: W_{m} \rightarrow \mathbf{R}^{m}
$$

given by

$$
u=\sum_{j=1}^{m} \xi_{j} e_{j} \mapsto S(u)=\xi=\left(\xi_{1}, \xi_{2}, \ldots, \xi_{m}\right)
$$

where

$$
|\xi|_{s}=\sum_{j=1}^{m}\left|\xi_{j}\right| \text { and } \quad\|u\|_{m}=\left(\int_{\Omega}|\nabla u|^{m} d x\right)^{\frac{1}{m}}
$$

Moreover,

$$
\|u\|_{m}=|\xi|_{s}=|S(u)|_{s}
$$

For each $m \in \mathbf{N}$, define the function $G: \mathbf{R}^{m} \rightarrow \mathbf{R}^{m}$ such that

$$
G(\xi)=G\left(\xi_{1}, \xi_{2}, \ldots, \xi_{m}\right)=\left(G_{1}(\xi), G_{2}(\xi), \ldots, G_{m}(\xi)\right),
$$

where $\xi=\left(\xi_{1}, \xi_{2}, \ldots, \xi_{m}\right) \in \mathbf{R}^{m}$,

$$
G_{j}(\xi)=\int_{\Omega} a_{0}\left(|\nabla u|^{p_{0}}\right) \mid \nabla u^{p_{0}-2} \nabla u \nabla e_{j} d x-\lambda_{0} \int_{\Omega} \frac{e_{j}}{(u+\varepsilon)^{\beta_{0}}} d x-\int_{\Omega} f_{0}(u) e_{j} d x,
$$

$j=1,2, \ldots, m$ and $u=\sum_{j=1}^{m} \xi_{j} e_{j} \in W_{m}$. Therefore,

$$
\langle G(\xi), \xi\rangle=\sum_{j=1}^{m} G_{j}(\xi) \xi_{j}=\int_{\Omega} a_{0}\left(|\nabla u|^{p_{0}}\right)|\nabla u|^{p_{0}} d x-\lambda_{0} \int_{\Omega} \frac{u}{(u+\varepsilon)^{\beta_{0}}} d x-\int_{\Omega} f_{0}(u) u d x .
$$

Note that

$$
\int_{\Omega} \frac{u}{(u+\varepsilon)^{\beta_{0}}} d x \leq|\Omega|
$$

Using (2.1) and Sobolev embedding, there exists positive constant $C_{1}$ such that

$$
\int_{\Omega} f_{0}(u) u d x \leq \delta C_{1}\|u\|_{1, p_{0}}^{p_{0}}+C_{\delta} \int_{\Omega}|u|^{q_{0}} \exp \left(\alpha|u|^{\frac{N}{N-1}}\right) d x
$$

Now, from $\left(a_{1}\right)$ we have

$$
\begin{aligned}
\int_{\Omega} a_{0}\left(|\nabla u|^{p_{0}}\right)|\nabla u|^{p_{0}} d x & \geq k_{1} \int_{\Omega}|\nabla u|^{p_{0}} d x+k_{2} \int_{\Omega}|\nabla u|^{N} d x \\
& =k_{1}\|u\|_{1, p_{0}}^{p_{0}}+k_{2}\|u\|_{1, N}^{N} .
\end{aligned}
$$

It follows from (3.3), (3.4) and (3.5) that

$$
\langle G(\xi), \xi\rangle \geq k_{2}\|u\|_{1, N}^{N}+\left(k_{1}-\delta C_{1}\right)\|u\|_{1, p_{0}}^{p_{0}}-\lambda_{0}|\Omega|-C_{\delta} \int_{\Omega}|u|^{q_{0}} \exp \left(\alpha|u|^{\frac{N}{N-1}}\right) d x
$$

Taking $\delta>0$ sufficiently small such that $\left(k_{1}-\delta C_{1}\right)>0$, we can rewrite $(3.6)$ as

$$
\langle G(\xi), \xi\rangle \geq k_{2}\|u\|_{1, N}^{N}-\lambda_{0}|\Omega|-C_{\delta} \int_{\Omega}|u|^{q_{0}} \exp \left(\alpha|u|^{\frac{N}{N-1}}\right) d x
$$


Using Hölder's inequality with $s, s^{\prime}>1$ such that $\frac{1}{s}+\frac{1}{s^{\prime}}=1$, we get

$$
C_{\delta} \int_{\Omega}|u|^{q_{0}} \exp \left(\alpha|u|^{\frac{N}{N-1}}\right) d x \leq C_{\delta}\left(\int_{\Omega}|u|^{q_{0} s^{\prime}} d x\right)^{\frac{1}{s^{\prime}}}\left(\int_{\Omega} \exp \left(\alpha s|u|^{\frac{N}{N-1}}\right) d x\right)^{\frac{1}{s}} .
$$

Since $q_{0}>N$ and $s^{\prime}>1$, by Sobolev embedding there exists $\widetilde{C_{1}}>0$ such that

$$
C_{\delta} \int_{\Omega}|u|^{q_{0}} \exp \left(\alpha|u|^{\frac{N}{N-1}}\right) d x \leq C_{\delta} \widetilde{C_{1}}\|u\|_{1, N}^{q_{0}}\left(\int_{\Omega} \exp \left(\alpha s|u|^{\frac{N}{N-1}}\right) d x\right)^{\frac{1}{s}} .
$$

Then, it follows (3.7) and (3.8) that

$$
\langle G(\xi), \xi\rangle \geq k_{2}\|u\|_{1, N}^{N}-\lambda_{0}|\Omega|-C_{\delta} \widetilde{C_{1}}\|u\|_{1, N}^{q_{0}}\left(\int_{\Omega} \exp \left(\alpha s|u|^{\frac{N}{N-1}}\right) d x\right)^{\frac{1}{s}} .
$$

Assume now that $\|u\|_{1, N}=r$ for some $r>0$ to be chosen later. We have

$$
\begin{aligned}
\int_{\Omega} \exp \left(\alpha s|u|^{\frac{N}{N-1}}\right) d x & =\int_{\Omega} \exp \left(\alpha s\|u\|_{1, N}^{\frac{N}{N-1}}\left(\frac{|u|}{\|u\|_{1, N}}\right)^{\frac{N}{N-1}}\right) d x \\
& =\int_{\Omega} \exp \left(\alpha s r^{\frac{N}{N-1}}\left(\frac{|u|}{\|u\|_{1, N}}\right)^{\frac{N}{N-1}}\right) d x
\end{aligned}
$$

and in order to apply the Theorem 2.1, we impose that

$$
r \leq\left(\frac{\alpha_{N}}{\alpha s}\right)^{\frac{N-1}{N}} .
$$

Therefore, there exists $M>0$ such that

$$
\sup _{\|u\|_{1, N} \leq 1} \int_{\Omega} \exp \left(\alpha s^{\frac{N}{N-1}}\left(\frac{|u|}{\|u\|_{1, N}}\right)\right) d x \leq M
$$

and hence,

$$
\langle G(\xi), \xi\rangle \geq k_{2} r^{N}-\lambda_{0}|\Omega|-C_{\delta} \widetilde{C_{1}} M^{1 / s} r^{q} .
$$

Now, it is necessary to choose $r$ such that

$$
k_{2} r^{N}-C_{\delta} \widetilde{C_{1}} M^{1 / s} r^{q} \geq \frac{k_{2} r^{N}}{2}
$$

in others words,

$$
r \leq\left(\frac{k_{2}}{2 C_{\delta} \widetilde{C}_{1} M^{\frac{1}{s}}}\right)^{\frac{1}{q-N}}
$$

Thus, considering $r=\min \left\{\left(\frac{\alpha_{N}}{\alpha s}\right)^{\frac{N-1}{N}},\left(\frac{k_{2}}{2 C_{\delta} \widetilde{C_{1}} M^{\frac{1}{s}}}\right)^{\frac{1}{q-N}}\right\}$ we get

$$
\langle G(\xi), \xi\rangle \geq \frac{k_{2} r^{N}}{2}-\lambda_{0}|\Omega| \text {. }
$$

Furthermore, choosing

we obtain

$$
\lambda^{*}=\frac{k_{2} r^{N}}{4|\Omega|}
$$

$$
\langle G(\xi), \xi\rangle>0, \text { for all } 0<\lambda_{0}<\lambda^{*}, \quad \xi \in \mathbf{R}^{m} \text { and }|\xi|_{s}=r \text {. }
$$


By virtue of Lemma 3.1, for every $m \in \mathbf{N}$, there exists $y \in \mathbf{R}^{m}$ with $|y|_{s} \leq r<1$ such that $G(y)=0$. Thus, from (3.2) there exists $u_{m} \in W_{m}$ satisfying

$$
\left\|u_{m}\right\|_{1, N} \leq r<1, \text { for every } \mathrm{m} \in \mathbf{N}
$$

such that

$$
\int_{\Omega} a_{0}\left(\left|\nabla u_{m}\right|^{p_{0}}\right)\left|\nabla u_{m}\right|^{p_{0}-2} \nabla u_{m} \nabla e_{j} d x=\lambda_{0} \int_{\Omega} \frac{e_{j}}{\left(u_{m}+\varepsilon\right)^{\beta_{0}}} d x+\int_{\Omega} f_{0}\left(u_{m}\right) e_{j} d x
$$

$j=1,2, \ldots, m$. Multiplying the equality (3.10) by any constant $\sigma_{j}$, for each $j=$ $1,2, \ldots, m$, and adding them, we conclude

$$
\int_{\Omega} a_{0}\left(\left|\nabla u_{m}\right|^{p_{0}}\right)\left|\nabla u_{m}\right|^{p_{0}-2} \nabla u_{m} \nabla \phi d x=\lambda_{0} \int_{\Omega} \frac{\phi}{\left(u_{m}+\varepsilon\right)^{\beta_{0}}} d x+\int_{\Omega} f_{0}\left(u_{m}\right) \phi d x,
$$

for all $\phi \in W_{m}$, which shows that $u_{m}$ is an approximate weak solution of problem (3.1).

Since $r$ does not depend on $m$ and $W_{m} \subset W_{0}^{1, N}(\Omega)$, for all $m \in \mathbf{N}$, then $\left(u_{m}\right)$ is a bounded sequence in $W_{0}^{1, N}(\Omega)$. Thus, for some subsequence, there exists $u \in$ $W_{0}^{1, N}(\Omega)$ such that

$$
\begin{cases}u_{m} \rightarrow u & \text { in } W_{0}^{1, N}(\Omega), \\ u_{m} \rightarrow u & \text { in } L^{\theta}(\Omega), \theta \geq 1 \\ u_{m}(x) \rightarrow u(x) & \text { a.e. in } \Omega \\ \left|u_{m}(x)\right| \leq g(x) \in L^{\theta}(\Omega) & \text { a.e. in } \Omega, \theta \geq 1 .\end{cases}
$$

Fix $k \in \mathbf{N}$ and consider $m \geq k$, then $W_{k} \subset W_{m}$ and

$$
\int_{\Omega} a_{0}\left(\left|\nabla u_{m}\right|^{p_{0}}\right)\left|\nabla u_{m}\right|^{p_{0}-2} \nabla u_{m} \nabla \phi_{k} d x=\lambda_{0} \int_{\Omega} \frac{\phi_{k}}{\left(u_{m}+\varepsilon\right)^{\beta_{0}}} d x+\int_{\Omega} f_{0}\left(u_{m}\right) \phi_{k} d x
$$

for all $\phi_{k} \in W_{k}$. Since $\phi_{k} \in W_{k}$, note that

$$
\left|\frac{\phi_{k}}{\left(u_{m}+\varepsilon\right)^{\beta_{0}}}\right| \leq \frac{\left|\phi_{k}\right|}{\varepsilon^{\beta_{0}}} \in L^{1}(\Omega)
$$

and by (3.12) we have

$$
\frac{\phi_{k}}{\left(u_{m}(x)+\varepsilon\right)^{\beta_{0}}} \rightarrow \frac{\phi_{k}}{(u(x)+\varepsilon)^{\beta_{0}}} \quad \text { a.e. in } \Omega \text {. }
$$

Therefore, we use [6, Theorem 4.2] to obtain that

$$
\int_{\Omega} \frac{\phi_{k}}{\left(u_{m}+\varepsilon\right)^{\beta_{0}}} d x \rightarrow \int_{\Omega} \frac{\phi_{k}}{(u+\varepsilon)^{\beta_{0}}} d x .
$$

Now, since $f_{0}$ is a continuous function, by (3.12) again we have

$$
f_{0}\left(u_{m}(x)\right) \phi_{k} \rightarrow f_{0}(u(x)) \phi_{k} \quad \text { a.e. in } \Omega \text {. }
$$

Using (2.1) we get

$$
\left|f_{0}\left(u_{m}(x)\right) \phi_{k}\right| \leq \delta\left|u_{m}(x)\right|^{p_{0}-1}\left|\phi_{k}\right|+C_{\delta}\left|u_{m}(x)\right|^{q_{0}-1} \exp \left(\alpha\left|u_{m}(x)\right|^{\frac{N}{N-1}}\right)\left|\phi_{k}\right| .
$$

We will need to prove that the function $\widehat{g}: \mathbf{R} \rightarrow \mathbf{R}$ defined by

$$
\widehat{g}\left(u_{m}(x)\right):=\delta\left|u_{m}(x)\right|^{p_{0}-1}\left|\phi_{k}\right|+C_{\delta}\left|u_{m}(x)\right|^{q_{0}-1} \exp \left(\alpha\left|u_{m}(x)\right|^{\frac{N}{N-1}}\right)\left|\phi_{k}\right|
$$

satisfies

$$
\left|f_{0}\left(u_{m}(x)\right) \phi_{k}\right| \leq \widehat{g}\left(u_{m}(x)\right) \in L^{1}(\Omega)
$$


It is sufficient to show that $\widehat{g}\left(u_{m}(x)\right)$ is convergent in $L^{1}(\Omega)$. Indeed, since $2 \leq$ $p_{0}<N$, we invoke (3.12) to obtain

$$
\left|u_{m}(x)\right|^{p_{0}-1}\left|\phi_{k}\right| \rightarrow|u(x)|^{p_{0}-1}\left|\phi_{k}\right| \quad \text { a.e. in } \Omega
$$

and

$$
\left|u_{m}(x)\right|^{p_{0}-1}\left|\phi_{k}\right| \leq g(x)^{p_{0}-1}\left|\phi_{k}\right| \in L^{1}(\Omega) .
$$

It follows from (3.17), (3.18) and [6, Theorem 4.2] that

$$
\int_{\Omega}\left|u_{m}\right|^{p_{0}-1}\left|\phi_{k}\right| d x \rightarrow \int_{\Omega}|u|^{p_{0}-1}\left|\phi_{k}\right| d x .
$$

Furthermore, from (3.12) again we get

$$
\left|u_{m}(x)\right|^{q_{0}-1} \exp \left(\alpha\left|u_{m}(x)\right|^{\frac{N}{N-1}}\right) \rightarrow|u(x)|^{q_{0}-1} \exp \left(\alpha|u(x)|^{\frac{N}{N-1}}\right) \quad \text { a.e. in } \Omega .
$$

Now, considering $s, s^{\prime}>1$ such that $\frac{1}{s}+\frac{1}{s^{\prime}}=1$, we use (3.12) and the fact that $q_{0}>N$ to obtain

$$
\left|u_{m}\right|^{q_{0}-1} \rightarrow|u|^{q_{0}-1} \quad \text { in } L^{s^{\prime}}(\Omega) .
$$

Moreover, by (3.9) we have

$$
\begin{aligned}
\int_{\Omega} \exp \left(\alpha s \mid u_{m}(x)^{\frac{N}{N-1}}\right) d x & =\int_{\Omega} \exp \left(\alpha s\left\|u_{m}(x)\right\|_{1, N}^{\frac{N}{N-1}}\left(\frac{\left|u_{m}(x)\right|}{\left\|u_{m}(x)\right\|_{1, N}}\right)^{\frac{N}{N-1}}\right) d x \\
& \leq \int_{\Omega} \exp \left(\alpha s r^{\frac{N}{N-1}}\left(\frac{\left|u_{m}(x)\right|}{\left\|u_{m}(x)\right\|_{1, N}}\right)^{\frac{N}{N-1}}\right) d x
\end{aligned}
$$

and applying the Theorem 2.1 we obtain

$$
\int_{\Omega} \exp \left(\alpha s\left|u_{m}(x)\right|^{\frac{N}{N-1}}\right) d x \leq \int_{\Omega} \exp \left(\alpha_{N}\left(\frac{\left|u_{m}(x)\right|}{\left\|u_{m}(x)\right\|_{1, N}}\right)^{\frac{N}{N-1}}\right) d x \leq M .
$$

Hence, by (3.21), (3.22) and Hölder's inequality we get

$$
\begin{aligned}
& \int_{\Omega}\left|u_{m}\right|^{q_{0}-1} \exp \left(\alpha\left|u_{m}\right|^{\frac{N}{N-1}}\right) d x \\
& \leq\left(\int_{\Omega}\left|u_{m}\right|^{\left(q_{0}-1\right) s^{\prime}} d x\right)^{\frac{1}{s^{\prime}}}\left(\int_{\Omega} \exp \left(\alpha s\left|u_{m}\right|^{\frac{N}{N-1}}\right) d x\right)^{\frac{1}{s}} \\
& \leq\left|u_{m}\right|_{L^{s^{\prime}}(\Omega)}^{q_{0}-1} M^{\frac{1}{s}}=\bar{M} .
\end{aligned}
$$

We use (3.20), (3.23) and [26, Theorem 4.8] to conclude that

$$
\left|u_{m}\right|^{q_{0}-1} \exp \left(\alpha\left|u_{m}\right|^{\frac{N}{N-1}}\right) \rightarrow|u|^{q_{0}-1} \exp \left(\alpha|u|^{\frac{N}{N-1}}\right) .
$$

It follows from (3.24) that

$$
\int_{\Omega}\left|u_{m}\right|^{q_{0}-1} \exp \left(\alpha\left|u_{m}\right|^{\frac{N}{N-1}}\right)\left|\phi_{k}\right| d x \rightarrow \int_{\Omega}|u|^{q_{0}-1} \exp \left(\alpha|u|^{\frac{N}{N-1}}\right)\left|\phi_{k}\right| d x .
$$

Therefore, by (3.19) and (3.25) we prove that

$$
\int_{\Omega} \widehat{g}\left(u_{m}(x)\right) d x \rightarrow \delta \int_{\Omega}|u(x)|^{p_{0}-1}\left|\phi_{k}\right| d x+C_{\delta} \int_{\Omega}|u(x)|^{q_{0}-1} \exp \left(\alpha|u(x)|^{\frac{N}{N-1}}\right)\left|\phi_{k}\right| d x,
$$

which shows the identity (3.16). 
Then, we use (3.15), (3.16) and [6, Theorem 4.2] to conclude that

$$
\int_{\Omega} f_{0}\left(u_{m}\right) \phi_{k} d x \rightarrow \int_{\Omega} f_{0}(u) \phi_{k} d x
$$

The next step is to show that

$$
\int_{\Omega} a_{0}\left(\left|\nabla u_{m}\right|^{p_{0}}\right)\left|\nabla u_{m}\right|^{p_{0}-2} \nabla u_{m} \nabla \phi_{k} d x \rightarrow \int_{\Omega} a_{0}\left(|\nabla u|^{p_{0}}\right)|\nabla u|^{p_{0}-2} \nabla u \nabla \phi_{k} d x
$$

To this end, we will use $\left(a_{2}\right)$ and the same reasoning in [18, Lemma 2.4] to obtain

$$
\begin{aligned}
0 & \leq C\left\|u_{m}-u\right\|_{1, N} \\
& \leq \int_{\Omega} a_{0}\left(\left|\nabla u_{m}\right|^{p_{0}}\right)\left|\nabla u_{m}\right|^{p_{0}} d x-\int_{\Omega} a_{0}\left(\left|\nabla u_{m}\right|^{p_{0}}\right)\left|\nabla u_{m}\right|^{p_{0}-2} \nabla u_{m} \nabla u d x+o_{n}(1) \\
& =\lambda_{0} \int_{\Omega} \frac{u_{m}}{\left(u_{m}+\varepsilon\right)^{\beta_{0}}} d x+\int_{\Omega} f_{0}\left(u_{m}\right) u_{m} d x-\lambda_{0} \int_{\Omega} \frac{u}{\left(u_{m}+\varepsilon\right)^{\beta_{0}}} d x-\int_{\Omega} f_{0}\left(u_{m}\right) u d x \\
& =o_{n}(1),
\end{aligned}
$$

where

$$
o_{n}(1)=\int_{\Omega} a_{0}\left(|\nabla u|^{p_{0}}\right)|\nabla u|^{p_{0}} d x-\int_{\Omega} a_{0}\left(|\nabla u|^{p_{0}}\right)|\nabla u|^{p_{0}-2} \nabla u_{m} \nabla u d x .
$$

Hence,

which implies that

$$
\left\|u_{m}-u\right\|_{1, N}=o_{n}(1)
$$

$$
u_{m} \rightarrow u \quad \text { in } W_{0}^{1, N}(\Omega) .
$$

Now, we know that the function defined by

$$
E(u)=\int_{\Omega} a_{0}\left(|\nabla u|^{p_{0}}\right)|\nabla u|^{p_{0}-2} \nabla u \nabla \phi_{k} d x
$$

is continuous. Then, we invoke this fact and (3.28) to get the convergence (3.27). Letting $m \rightarrow \infty$ in (3.13), we use (3.14), (3.26) and (3.27) to conclude that

$$
\int_{\Omega} a_{0}\left(|\nabla u|^{p_{0}}\right)|\nabla u|^{p_{0}-2} \nabla u \nabla \phi_{k} d x=\lambda_{0} \int_{\Omega} \frac{\phi_{k}}{(u+\varepsilon)^{\beta_{0}}} d x+\int_{\Omega} f_{0}(u) \phi_{k} d x
$$

for all $\phi_{k} \in W_{k}$.

Since $\left[W_{k}\right]_{k \in \mathbf{N}}$ is dense in $W_{0}^{1, N}(\Omega)$, we have

$$
\phi_{k} \rightarrow \phi \quad \text { as } k \rightarrow \infty
$$

Then,

$$
\begin{aligned}
\int_{\Omega} a_{0}\left(|\nabla u|^{p_{0}}\right)|\nabla u|^{p_{0}-2} \nabla u \nabla \phi_{k} d x & \rightarrow \int_{\Omega} a_{0}\left(|\nabla u|^{p_{0}}\right)|\nabla u|^{p_{0}-2} \nabla u \nabla \phi d x, \\
\int_{\Omega} \frac{\phi_{k}}{(u+\varepsilon)^{\beta_{0}}} d x & \rightarrow \int_{\Omega} \frac{\phi}{(u+\varepsilon)^{\beta_{0}}} d x
\end{aligned}
$$

and

$$
\int_{\Omega} f_{0}(u) \phi_{k} d x \rightarrow \int_{\Omega} f_{0}(u) \phi d x .
$$

Therefore, since $\phi \in W_{0}^{1, N}(\Omega)$ is arbitrary, it follows from (3.29) - (3.32) that

$$
\int_{\Omega} a_{0}\left(|\nabla u|^{p_{0}}\right)|\nabla u|^{p_{0}-2} \nabla u \nabla \phi d x=\lambda_{0} \int_{\Omega} \frac{\phi}{(u+\varepsilon)^{\beta_{0}}} d x+\int_{\Omega} f_{0}(u) \phi d x,
$$


for all $\phi \in W_{0}^{1, N}(\Omega)$, which shows that $u$ is a weak solution of the problem (3.1). Furthermore, $u>0$ in $\Omega$. In fact, since $f_{0}(t)=0, \forall t<0$, we use $\phi=u^{-}$in (3.33) to obtain

$$
\int_{\Omega} a_{0}\left(|\nabla u|^{p_{0}}\right)\left|\nabla u^{-}\right| d x \leq 0 .
$$

It follows from $\left(a_{1}\right)$ and $k_{2}>0$ that

$$
\int_{\Omega}\left|\nabla u^{-}\right|^{N}=\left\|u^{-}\right\|_{1, N}^{N}=0
$$

which implies that $u^{-}=0$ and then $u=u^{+} \geq 0$. But thanks to the Harnack's inequality, see [21], $u>0$ in $\Omega$.

\section{Proof of the Theorem 1.1}

For each $n \in \mathbf{N}$, let $\varepsilon=\frac{1}{n}$ and $u_{\frac{1}{n}}=u_{n}$ be, where $u_{n}$ is a solution of auxiliary problem (3.1)

$$
\begin{cases}-\operatorname{div}\left(a_{0}\left(\left|\nabla u_{n}\right|^{p_{0}}\right)\left|\nabla u_{n}\right|^{p_{0}-2} \nabla u_{n}\right)=\frac{\lambda_{0}}{\left(u_{n}+\frac{1}{n}\right)^{\beta_{0}}}+f_{0}\left(u_{n}\right) & \text { in } \Omega, \\ u_{n}>0 & \text { in } \Omega, \\ u_{n}=0 & \text { on } \partial \Omega,\end{cases}
$$

obtained by the Lemma 3.2. Note that, from $\left(f_{3}\right)$ we get

$$
\frac{\lambda_{0}}{\left(u_{n}+\frac{1}{n}\right)^{\beta_{0}}}+f_{0}\left(u_{n}\right) \geq \frac{\lambda_{0}}{\left(u_{n}+1\right)^{\beta_{0}}}+\left|u_{n}\right|^{\gamma_{0}-1}
$$

Since the function $t \mapsto \frac{\lambda_{0}}{(t+1)^{\beta_{0}}}+t^{\gamma_{0}-1}$, for all $t \geq 0$, attains a positive minimum $z$. Then,

$$
-\operatorname{div}\left(a_{0}\left(\left|\nabla u_{n}\right|^{p_{0}}\right)\left|\nabla u_{n}\right|^{p_{0}-2} \nabla u_{n}\right) \geq z>0 \quad \text { in } \Omega .
$$

By virtue from Minty-Browder's Theorem [6, Theorem 5.15], we use the unique positive solution of the problem

$$
\begin{cases}-\operatorname{div}\left(a_{0}\left(|\nabla v|^{p_{0}}\right)|\nabla v|^{p_{0}-2} \nabla v\right)=z & \text { in } \Omega \\ v>0 & \text { in } \Omega \\ v=0 & \text { on } \partial \Omega\end{cases}
$$

to obtain

$$
\begin{cases}-\operatorname{div}\left(a_{0}\left(\left|\nabla u_{n}\right|^{p_{0}}\right)\left|\nabla u_{n}\right|^{p_{0}-2} \nabla u_{n}\right) \geq-\operatorname{div}\left(a_{0}\left(|\nabla v|^{p_{0}}\right)|\nabla v|^{p_{0}-2} \nabla v\right) & \text { in } \Omega, \\ u=v & \text { on } \partial \Omega .\end{cases}
$$

Hence, we use the Lemma 2.1 to conclude that

$$
u_{n}(x) \geq v(x)>0 \text { in } \Omega, \quad \forall n \in \mathbf{N},
$$

which implies that $u_{n}(x) \nrightarrow 0$, for each $x \in \Omega$.

Now, from (3.12) we get

$$
u_{m} \rightarrow u_{n} \text { in } W_{0}^{1, N}(\Omega) \text { as } m \rightarrow+\infty
$$

and it follows from (3.9) that

$$
\left\|u_{n}\right\|_{1, N} \leq \liminf _{m \rightarrow+\infty}\left\|u_{m}\right\|_{1, N} \leq r<1, \quad \text { for all } n \in \mathbf{N} .
$$


Therefore, $r$ does not depend on $n$, which shows that $\left(u_{n}\right)$ is a bounded sequence. Thus, since $W_{0}^{1, N}(\Omega)$ is a reflective Banach space, for some subsequence, there exists $u \in W_{0}^{1, N}(\Omega)$ such that

$$
\begin{cases}u_{n} \rightarrow u & \text { in } W_{0}^{1, N}(\Omega), \\ u_{n} \rightarrow u & \text { in } L^{\theta}(\Omega), \theta \geq 1, \\ u_{n}(x) \rightarrow u(x) & \text { a.e. in } \Omega \\ \left|u_{n}(x)\right| \leq g(x) \in L^{\theta}(\Omega) & \text { a.e. in } \Omega, \theta \geq 1 .\end{cases}
$$

Recall from (3.33) that

$$
\begin{aligned}
& \int_{\Omega} a_{0}\left(\left|\nabla u_{n}\right|^{p_{0}}\right)\left|\nabla u_{n}\right|^{p_{0}-2} \nabla u_{n} \nabla \phi d x \\
& =\lambda_{0} \int_{\Omega} \frac{\phi}{\left(u_{n}+\frac{1}{n}\right)^{\beta_{0}}} d x+\int_{\Omega} f_{0}\left(u_{n}\right) \phi d x, \quad \forall \phi \in W_{0}^{1, N}(\Omega) .
\end{aligned}
$$

Since $f_{0}$ is a continuous function, by $(4.3)$ we have

$$
f_{0}\left(u_{n}(x)\right) \phi \rightarrow f_{0}(u(x)) \phi \text { a.e. in } \Omega \text {. }
$$

By same computation in (3.16), we obtain the function $\widehat{g}: \mathbf{R} \rightarrow \mathbf{R}$ such that

$$
\left|f\left(u_{n}(x)\right) \phi\right| \leq \widehat{g}\left(u_{n}(x)\right)
$$

such that $\widehat{g}\left(u_{n}(x)\right)$ converges in $L^{1}(\Omega)$. Then, we use [6, Theorem 4.2] to conclude that

$$
\int_{\Omega} f_{0}\left(u_{n}\right) \phi d x \rightarrow \int_{\Omega} f_{0}(u) \phi d x, \quad \forall \phi \in W_{0}^{1, N}(\Omega)
$$

Now, by same computation in (3.27), we have

$$
\int_{\Omega} a_{0}\left(\left|\nabla u_{n}\right|^{p_{0}}\right)\left|\nabla u_{n}\right|^{p_{0}-2} \nabla u_{n} \nabla \phi d x \rightarrow \int_{\Omega} a_{0}\left(|\nabla u|^{p_{0}}\right)|\nabla u|^{p_{0}-2} \nabla u \nabla \phi d x,
$$

for all $\phi \in W_{0}^{1, N}(\Omega)$. Note that, from (4.3) again we get

$$
\frac{\phi}{\left(u_{n}(x)+\frac{1}{n}\right)^{\beta_{0}}} \rightarrow \frac{\phi}{u(x)^{\beta_{0}}} \quad \text { a.e. in } \Omega \text {. }
$$

Moreover, by virtue from (4.1) and $\left(a_{1}\right)$, we can argue as in [23] to obtain that $v \in C^{1}(\bar{\Omega})$. Consequently, from (4.2), for each $x \in \Omega$, it follows that $u_{n}(x) \geq v(x)>$ $C d(x)>0$, where $d(x)=\operatorname{dist}(x, \partial \Omega)$ and $C$ is a positive constant that does not depend on $x$. Thus,

$$
\int_{\Omega} \frac{\phi}{\left(u_{n}(x)+\frac{1}{n}\right)^{\beta_{0}}} d x \leq \int_{\Omega} \frac{\phi}{u_{n}(x)^{\beta_{0}}} d x \leq \int_{\Omega} \frac{\phi}{C d(x)^{\beta_{0}}} d x .
$$

Hence, we invoke Theorem 2.2 to obtain $\left|\frac{\phi}{C d(x)^{\beta_{0}}}\right| \in L^{r}(\Omega)$ and $C_{2}>0$ such that

$$
\int_{\Omega} \frac{\phi}{\left(u_{n}(x)+\frac{1}{n}\right)^{\beta_{0}}} d x \leq C_{2}\|\phi\|_{1, N} .
$$

Therefore, by (4.7), (4.8) and [6, Theorem 4.2] we get

$$
\int_{\Omega} \frac{\phi}{\left(u_{n}+\frac{1}{n}\right)^{\beta_{0}}} d x \rightarrow \int_{\Omega} \frac{\phi}{u^{\beta_{0}}} d x, \quad \forall \phi \in W_{0}^{1, N}(\Omega) .
$$


Letting $n \rightarrow+\infty$ in (4.4), we use (4.5), (4.6) and (4.9) to conclude that

$$
\int_{\Omega} a_{0}\left(|\nabla u|^{p_{0}}\right)|\nabla u|^{p_{0}-2} \nabla u \nabla \phi d x=\lambda_{0} \int_{\Omega} \frac{\phi}{u^{\beta_{0}}} d x+\int_{\Omega} f_{0}(u) \phi d x, \quad \forall \phi \in W_{0}^{1, N}(\Omega),
$$

which proves that $u \in W_{0}^{1, N}(\Omega)$ is a weak solution for the problem (1.1).

\section{Auxiliary problem for the case system}

For each $\varepsilon>0$, we consider the following auxiliary problem

$$
\begin{cases}-\operatorname{div}\left(a_{1}\left(|\nabla u|^{p_{1}}\right)|\nabla u|^{p_{1}-2} \nabla u\right)=\frac{\lambda_{1}}{(u+\varepsilon)^{\beta_{1}}}+f_{1}(v) & \text { in } \Omega, \\ -\operatorname{div}\left(a_{2}\left(|\nabla v|^{p_{2}}\right)|\nabla v|^{p_{2}-2} \nabla v\right)=\frac{\lambda_{2}}{(v+\varepsilon)^{\beta_{2}}}+f_{2}(u) & \text { in } \Omega, \\ u, v>0 & \text { in } \Omega, \\ u=v=0 & \text { on } \partial \Omega,\end{cases}
$$

where the functions $a_{i}$ and $f_{i}(i=1,2)$ satisfy the hypotheses of the Theorem 1.2.

The main result in this section is the following:

Lemma 5.1. For each $0<\varepsilon<1$, there exists $\lambda^{*}>0$ such that the problem (5.1) has a weak positive solution for every $0<\lambda_{1}+\lambda_{2}<\lambda^{*}$, with $i=1,2$.

Proof. Let $B=\left\{e_{1}, e_{2}, \ldots, e_{m}, \ldots\right\}$ be a Schauder basis of $W_{0}^{1, N}(\Omega)$. For each $m \in \mathbf{N}$, define

$$
W_{m}=\left[e_{1}, e_{2}, \ldots, e_{m}\right]
$$

to be the finite-dimensional space generated by $\left\{e_{1}, e_{2}, \ldots, e_{m}\right\}$.

For each $m \in \mathbf{N}$, we define the function $J: \mathbf{R}^{2 m} \rightarrow \mathbf{R}^{2 m}$ such that

$$
J(\eta, \xi)=\left(F_{1}(\eta, \xi), F_{2}(\eta, \xi), \ldots, F_{m}(\eta, \xi), G_{1}(\eta, \xi), G_{2}(\eta, \xi), \ldots, G_{m}(\eta, \xi)\right),
$$

where $(\eta, \xi)=\left(\eta_{1}, \eta_{2}, \ldots, \eta_{m}, \xi_{1}, \xi_{2}, \ldots, \xi_{m}\right) \in \mathbf{R}^{2 m}$,

$$
F_{j}(\eta, \xi)=\int_{\Omega} a_{1}\left(|\nabla u|^{p_{1}}\right)|\nabla u|^{p_{1}-2} \nabla u \nabla e_{j} d x-\lambda_{1} \int_{\Omega} \frac{e_{j}}{(u+\varepsilon)^{\beta_{1}}} d x-\int_{\Omega} f_{1}(v) e_{j} d x,
$$

$j=1,2, \ldots, m$,

$$
G_{j}(\eta, \xi)=\int_{\Omega} a_{2}\left(|\nabla v|^{p_{2}}\right)|\nabla v|^{p_{2}-2} \nabla v \nabla e_{j} d x-\lambda_{2} \int_{\Omega} \frac{e_{j}}{(v+\varepsilon)^{\beta_{2}}} d x-\int_{\Omega} f_{2}(u) e_{j} d x,
$$

$j=1,2, \ldots, m$,

$$
u=\sum_{j=1}^{m} \eta_{j} e_{j} \in W_{m}
$$

and

$$
v=\sum_{j=1}^{m} \xi_{j} e_{j} \in W_{m}
$$

Moreover,

$$
\|(u, v)\|=|(\eta, \xi)|_{s}
$$


Therefore,

$$
\begin{aligned}
\langle J(\eta, \xi),(\eta, \xi)\rangle= & \left\langle\left(F_{1}(\eta, \xi), F_{2}(\eta, \xi), \ldots, F_{m}(\eta, \xi), G_{1}(\eta, \xi), G_{2}(\eta, \xi), \ldots, G_{m}(\eta, \xi)\right),\right. \\
& \left.\left(\eta_{1}, \eta_{2}, \ldots, \eta_{m}, \xi_{1}, \xi_{2}, \ldots, \xi_{m}\right)\right\rangle=\sum_{j=1}^{m} F_{j}(\eta, \xi) \eta_{j}+\sum_{j=1}^{m} G_{j}(\eta, \xi) \xi_{j} \\
= & \int_{\Omega} a_{1}\left(|\nabla u|^{p_{1}}\right)|\nabla u|^{p_{1}} d x-\lambda_{1} \int_{\Omega} \frac{u}{(u+\varepsilon)^{\beta_{1}}} d x-\int_{\Omega} f_{1}(v) u d x \\
& +\int_{\Omega} a_{2}\left(|\nabla v|^{p_{2}}\right)|\nabla v|^{p_{2}} d x-\lambda_{2} \int_{\Omega} \frac{v}{(v+\varepsilon)^{\beta_{2}}} d x-\int_{\Omega} f_{2}(u) v d x .
\end{aligned}
$$

Note that

$$
\int_{\Omega} \frac{u}{(u+\varepsilon)^{\beta_{1}}} d x \leq|\Omega|
$$

and

$$
\int_{\Omega} \frac{v}{(v+\varepsilon)^{\beta_{2}}} d x \leq|\Omega|
$$

Using (2.1), Young's inequality and Sobolev embedding, there exist positive constants $C_{1}, C_{2}, C_{3}, C_{4}, C_{5}, C_{6}, C_{7}$ and $C_{8}$ such that

$$
\begin{aligned}
\int_{\Omega} f_{1}(v) u d x \leq & \delta_{1} C_{1}\|v\|_{1, p_{1}}^{p_{1}}+\delta_{1} C_{2}\|u\|_{1, p_{1}}^{p_{1}}+C_{\delta_{1}} C_{3} \int_{\Omega}|v|^{q_{1}} \exp \left(\alpha_{1}|v|^{\frac{N}{N-1}}\right) d x \\
& +C_{\delta_{1}} C_{4} \int_{\Omega}|u|^{q_{1}} \exp \left(\alpha_{1}|v|^{\frac{N}{N-1}}\right) d x
\end{aligned}
$$

and

$$
\begin{aligned}
\int_{\Omega} f_{2}(u) v d x \leq & \delta_{2} C_{5}\|u\|_{1, p_{2}}^{p_{2}}+\delta_{2} C_{6}\|v\|_{1, p_{2}}^{p_{2}}+C_{\delta_{2}} C_{7} \int_{\Omega}|u|^{q_{2}} \exp \left(\alpha_{2}|u|^{\frac{N}{N-1}}\right) d x \\
& +C_{\delta_{2}} C_{8} \int_{\Omega}|v|^{q_{2}} \exp \left(\alpha_{2}|u|^{\frac{N}{N-1}}\right) d x
\end{aligned}
$$

Now, from $\left(a_{1}\right)$ we have

$$
\begin{aligned}
\int_{\Omega} a_{1}\left(|\nabla u|^{p_{1}}\right)|\nabla u|^{p_{1}} d x & \geq k_{1} \int_{\Omega}|\nabla u|^{p_{1}} d x+k_{2} \int_{\Omega}|\nabla u|^{N} d x \\
& =k_{1}\|u\|_{1, p_{1}}^{p_{1}}+k_{2}\|u\|_{1, N}^{N}
\end{aligned}
$$

and

$$
\begin{aligned}
\int_{\Omega} a_{2}\left(|\nabla v|^{p_{2}}\right)|\nabla v|^{p_{2}} d x & \geq k_{1} \int_{\Omega}|\nabla u|^{p_{2}} d x+k_{2} \int_{\Omega}|\nabla v|^{N} d x \\
& =k_{1}\|v\|_{1, p_{2}}^{p_{2}}+k_{2}\|v\|_{1, N}^{N} .
\end{aligned}
$$

It follows from (5.3)-(5.8) that

$$
\begin{aligned}
\langle J(\eta, \xi),(\eta, \xi)\rangle \geq k_{2}\left(\|u\|_{1, N}^{N}+\|v\|_{1, N}^{N}\right)+\left(k_{1}-\delta_{1} C_{2}\right)\|u\|_{1, p_{1}}^{p_{1}}+\left(k_{1}-\delta_{2} C_{6}\right)\|v\|_{1, p_{2}}^{p_{2}} \\
-\left(\lambda_{1}+\lambda_{2}\right)|\Omega|-\delta_{1} C_{1}\|v\|_{1, p_{1}}^{p_{1}}-C_{\delta_{1}} C_{3} \int_{\Omega}|v|^{q_{1}} \exp \left(\alpha_{1}|v|^{\frac{N}{N-1}}\right) d x \\
-C_{\delta_{1}} C_{4} \int_{\Omega}|u|^{q_{1}} \exp \left(\alpha_{1}|v|^{\frac{N}{N-1}}\right) d x-\delta_{2} C_{5}\|u\|_{1, p_{2}}^{p_{2}} \\
-C_{\delta_{2}} C_{7} \int_{\Omega}|u|^{q_{2}} \exp \left(\alpha_{2}|u|^{\frac{N}{N-1}}\right) d x-C_{\delta_{2}} C_{8} \int_{\Omega}|v|^{q_{2}} \exp \left(\alpha_{2}|u|^{\frac{N}{N-1}}\right) d x .
\end{aligned}
$$


Note that, from $2 \leq p_{1}, p_{2}<N$ and Sobolev embedding, there exist $C_{9}, C_{10}>0$ we have

and

$$
\delta_{1} C_{1}\|v\|_{1, p_{1}}^{p_{1}} \leq \delta_{1} C_{9}\|v\|_{1, N}^{p_{1}}
$$

$$
\delta_{2} C_{5}\|u\|_{1, p_{2}}^{p_{2}} \leq \delta_{2} C_{10}\|u\|_{1, N}^{p_{2}} .
$$

Since $\|(u, v)\|_{1, N}^{N}=\|u\|_{1, N}^{N}+\|v\|_{1, N}^{N}$ and taking $\delta_{1}, \delta_{2}>0$ sufficiently small such that $\left(k_{1}-\delta_{1} C_{2}\right),\left(k_{1}-\delta_{2} C_{6}\right)>0$ we obtain

$$
\begin{array}{r}
\langle J(\eta, \xi),(\eta, \xi)\rangle \geq k_{2}\|(u, v)\|^{N}-\left(\lambda_{1}+\lambda_{2}\right)|\Omega|-\delta_{1} C_{9}\|(u, v)\|_{1, N}^{p_{1}}-\delta_{2} C_{10}\|(u, v)\|_{1, N}^{p_{2}} \\
\quad-C_{\delta_{1}} C_{3} \int_{\Omega}|v|^{q_{1}} \exp \left(\alpha_{1}|v|^{\frac{N}{N-1}}\right) d x-C_{\delta_{1}} C_{4} \int_{\Omega}|u|^{q_{1}} \exp \left(\alpha_{1}|v|^{\frac{N}{N-1}}\right) d x \\
\quad-C_{\delta_{2}} C_{7} \int_{\Omega}|u|^{q_{2}} \exp \left(\alpha_{2}|u|^{\frac{N}{N-1}}\right) d x-C_{\delta_{2}} C_{8} \int_{\Omega}|v|^{q_{2}} \exp \left(\alpha_{2}|u|^{\frac{N}{N-1}}\right) d x .
\end{array}
$$

Using Hölder's inequality with $s, s^{\prime}>1$ such that $\frac{1}{s}+\frac{1}{s^{\prime}}=1$, we get

$$
\begin{aligned}
& C_{\delta_{1}} C_{3} \int_{\Omega}|v|^{q_{1}} \exp \left(\alpha_{1}|v|^{\frac{N}{N-1}}\right) d x \leq C_{\delta_{1}} C_{3}\left(\int_{\Omega}|v|^{q_{1} s^{\prime}} d x\right)^{\frac{1}{s^{\prime}}}\left(\int_{\Omega} \exp \left(\alpha_{1} s|v|^{\frac{N}{N-1}}\right) d x\right)^{\frac{1}{s}}, \\
& C_{\delta_{1}} C_{4} \int_{\Omega}|u|^{q_{1}} \exp \left(\alpha_{1}|v|^{\frac{N}{N-1}}\right) d x \leq C_{\delta_{1}} C_{4}\left(\int_{\Omega}|u|^{q_{1} s^{\prime}} d x\right)^{\frac{1}{s^{\prime}}}\left(\int_{\Omega} \exp \left(\alpha_{1} s|v|^{\frac{N}{N-1}}\right) d x\right)^{\frac{1}{s}}, \\
& C_{\delta_{2}} C_{7} \int_{\Omega}|u|^{q_{2}} \exp \left(\alpha_{2}|u|^{\frac{N}{N-1}}\right) d x \leq C_{\delta_{2}} C_{7}\left(\int_{\Omega}|u|^{q_{2} s^{\prime}} d x\right)^{\frac{1}{s^{\prime}}}\left(\int_{\Omega} \exp \left(\alpha_{2} s|u|^{\frac{N}{N-1}}\right) d x\right)^{\frac{1}{s}}
\end{aligned}
$$

and

$$
C_{\delta_{2}} C_{8} \int_{\Omega}|v|^{q_{2}} \exp \left(\alpha_{2}|u|^{\frac{N}{N-1}}\right) d x \leq C_{\delta_{2}} C_{8}\left(\int_{\Omega}|v|^{q_{2} s^{\prime}} d x\right)^{\frac{1}{s^{\prime}}}\left(\int_{\Omega} \exp \left(\alpha_{2} s|u|^{\frac{N}{N-1}}\right) d x\right)^{\frac{1}{s}} .
$$

Since $q_{1}, q_{2}>N$ and $s^{\prime}>1$, by Sobolev embedding there exist $C_{11}, C_{12}, C_{13}, C_{14}>$ 0 such that

$$
\begin{aligned}
& C_{\delta_{1}} C_{3} \int_{\Omega}|v|^{q_{1}} \exp \left(\alpha_{1}|v|^{\frac{N}{N-1}}\right) d x \leq C_{\delta_{1}} C_{11}\|v\|_{1, N}^{q_{1}}\left(\int_{\Omega} \exp \left(\alpha_{1} s|v|^{\frac{N}{N-1}}\right) d x\right)^{\frac{1}{s}}, \\
& C_{\delta_{1}} C_{4} \int_{\Omega}|u|^{q_{1}} \exp \left(\alpha_{1}|v|^{\frac{N}{N-1}}\right) d x \leq C_{\delta_{1}} C_{12}\|u\|_{1, N}^{q_{1}}\left(\int_{\Omega} \exp \left(\alpha_{1} s|v|^{\frac{N}{N-1}}\right) d x\right)^{\frac{1}{s}}, \\
& C_{\delta_{2}} C_{7} \int_{\Omega}|u|^{q_{2}} \exp \left(\alpha_{2}|u|^{\frac{N}{N-1}}\right) d x \leq C_{\delta_{2}} C_{13}\|u\|_{1, N}^{q_{2}}\left(\int_{\Omega} \exp \left(\alpha_{2} s|u|^{\frac{N}{N-1}}\right) d x\right)^{\frac{1}{s}}
\end{aligned}
$$

and

$$
C_{\delta_{2}} C_{8} \int_{\Omega}|v|^{q_{2}} \exp \left(\alpha_{2}|u|^{\frac{N}{N-1}}\right) d x \leq C_{\delta_{2}} C_{14}\|v\|_{1, N}^{q_{2}}\left(\int_{\Omega} \exp \left(\alpha_{2} s|u|^{\frac{N}{N-1}}\right) d x\right)^{\frac{1}{s}} .
$$

Then, it follows from (5.9) that

$$
\begin{aligned}
\langle J(\eta, \xi),(\eta, \xi)\rangle \geq & k_{2}\|(u, v)\|^{N}-\left(\lambda_{1}+\lambda_{2}\right)|\Omega|-\delta_{1} C_{9}\|(u, v)\|_{1, N}^{p_{1}}-\delta_{2} C_{10}\|(u, v)\|_{1, N}^{p_{2}} \\
& -C_{\delta_{1}} C_{11}\|(u, v)\|_{1, N}^{q_{1}}\left(\int_{\Omega} \exp \left(\alpha_{1} s|v|^{\frac{N}{N-1}}\right) d x\right)^{\frac{1}{s}} \\
& -C_{\delta_{1}} C_{12}\|(u, v)\|_{1, N}^{q_{1}}\left(\int_{\Omega} \exp \left(\alpha_{1} s|v|^{\frac{N}{N-1}}\right) d x\right)^{\frac{1}{s}}
\end{aligned}
$$




$$
\begin{aligned}
& -C_{\delta_{2}} C_{13}\|(u, v)\|_{1, N}^{q_{2}}\left(\int_{\Omega} \exp \left(\alpha_{2} s|u|^{\frac{N}{N-1}}\right) d x\right)^{\frac{1}{s}} \\
& -C_{\delta_{2}} C_{14}\|(u, v)\|_{1, N}^{q_{2}}\left(\int_{\Omega} \exp \left(\alpha_{2} s|u|^{\frac{N}{N-1}}\right) d x\right)^{\frac{1}{s}} .
\end{aligned}
$$

Assume now that $\|(u, v)\|_{1, N}=r$ for some $r>0$ to be chosen later. Then, we have

$$
\begin{aligned}
\int_{\Omega} \exp \left(\alpha_{1} s|u|^{\frac{N}{N-1}}\right) d x & =\int_{\Omega} \exp \left(\alpha_{1} s\|u\|_{1, N}^{\frac{N}{N-1}}\left(\frac{|u|}{\|u\|_{1, N}}\right)^{\frac{N}{N-1}}\right) d x \\
& \leq \int_{\Omega} \exp \left(\alpha_{1} s \|(u, v)^{\frac{N}{N-1}}\left(\frac{|u|}{\|u\|_{1, N}}\right)^{\frac{N}{N-1}}\right) d x \\
& =\int_{\Omega} \exp \left(\alpha_{1} s r^{\frac{N}{N-1}}\left(\frac{|u|}{\|u\|_{1, N}}\right)^{\frac{N}{N-1}}\right) d x
\end{aligned}
$$

Similarly,

$$
\int_{\Omega} \exp \left(\alpha_{2} s|v|^{\frac{N}{N-1}}\right) d x \leq \int_{\Omega} \exp \left(\alpha_{2} s r^{\frac{N}{N-1}}\left(\frac{|v|}{\|v\|_{1, N}}\right)^{\frac{N}{N-1}}\right) d x
$$

and in order to apply the Theorem 2.1, we impose that

$$
r \leq\left(\frac{\alpha_{N}}{\alpha_{1} s}\right)^{\frac{N-1}{N}} \text { and } r \leq\left(\frac{\alpha_{N}}{\alpha_{2} s}\right)^{\frac{N-1}{N}} .
$$

Therefore, there exist $M_{1}, M_{2}>0$ such that

$$
\sup _{\|u\|_{1, N} \leq 1} \int_{\Omega} \exp \left(\alpha_{1} s r^{\frac{N}{N-1}}\left(\frac{|u|}{\|u\|_{1, N}}\right)\right) d x \leq M_{1}
$$

and

$$
\sup _{\|v\|_{1, N} \leq 1} \int_{\Omega} \exp \left(\alpha_{2} s r^{\frac{N}{N-1}}\left(\frac{|v|}{\|v\|_{1, N}}\right)\right) d x \leq M_{2} .
$$

Hence, there exist $C_{15}, C_{16}>0$ such that we can rewrite (5.10) as

$$
\begin{aligned}
& \langle J(\eta, \xi),(\eta, \xi)\rangle \\
& \geq k_{2} r^{N}-\left(\lambda_{1}+\lambda_{2}\right)|\Omega|-\delta_{1} C_{9} r^{p_{1}}-\delta_{2} C_{10} r^{p_{2}}-C_{\delta_{1}} C_{15} M_{2}^{1 / s} r^{q_{1}}-C_{\delta_{2}} C_{16} M_{1}^{1 / s} r^{q_{2}} .
\end{aligned}
$$

Now, it is necessary to choose $r$ such that

$$
\frac{k_{2} r^{N}}{2}-C_{\delta_{1}} \widetilde{C_{1}} M_{1}^{1 / s} r^{q_{1}} \geq \frac{k_{2} r^{N}}{4}
$$

and

in others words

$$
\frac{k_{2} r^{N}}{2}-C_{\delta_{2}} \widetilde{C_{2}} M_{2}^{1 / s} r^{q_{2}} \geq \frac{k_{2} r^{N}}{4}
$$

$$
r \leq\left(\frac{k_{2}}{4 C_{\delta_{1}} \widetilde{C_{1}} M_{1}^{\frac{1}{s}}}\right)^{\frac{1}{q_{1}-N}} \text { and } r \leq\left(\frac{k_{2}}{4 C_{\delta_{2}} \widetilde{C_{2}} M_{2}^{\frac{1}{s}}}\right)^{\frac{1}{q_{2}-N}} .
$$


Considering

$$
r=\min \left\{1,\left(\frac{\alpha_{N}}{\alpha_{1} s}\right)^{\frac{N-1}{N}},\left(\frac{\alpha_{N}}{\alpha_{2} s}\right)^{\frac{N-1}{N}},\left(\frac{k_{2}}{4 C_{\delta_{1}} \widetilde{C_{1}} M_{1}^{\frac{1}{s}}}\right)^{\frac{1}{q_{1}-N}},\left(\frac{k_{2}}{4 C_{\delta_{2}} \widetilde{C_{2}} M_{2}^{\frac{1}{s}}}\right)^{\frac{1}{q_{2}-N}}\right\},
$$

we get

$$
\langle J(\eta, \xi),(\eta, \xi)\rangle \geq k_{2} r^{N}-\left(\lambda_{1}+\lambda_{2}\right)|\Omega|-\delta_{1} C_{9}-\delta_{2} C_{10}
$$

Thus, since $r>0$ is fixed and the last inequality is true for all $\delta_{1}, \delta_{2}>0$ then, there exists $\lambda^{*}>0$ such that

$$
\langle J(\xi), \xi\rangle>0 \text {, for all } \eta, \xi \in \mathbf{R}^{m} \text { and }|(\eta, \xi)|_{s}=r, \text { for all } 0<\lambda_{1}+\lambda_{2}<\lambda^{*} \text {. }
$$

By virtue of Lemma 3.1, for every $m \in \mathbf{N}$, there exists $(x, y) \in \mathbf{R}^{2 m}$ with $|(x, y)|_{s} \leq r<1$ such that $J(x, y)=0$. Consequently, there exist $u_{m}, v_{m} \in W_{m}$ satisfying

$$
\left\|\left(u_{m}, v_{m}\right)\right\| \leq r<1, \text { for every } \mathrm{m} \in \mathbf{N}
$$

such that

$$
\int_{\Omega} a_{1}\left(\left|\nabla u_{m}\right|^{p_{1}}\right)\left|\nabla u_{m}\right|^{p_{1}-2} \nabla u_{m} \nabla \phi d x=\lambda_{1} \int_{\Omega} \frac{\phi}{\left(u_{m}+\varepsilon\right)^{\beta_{1}}} d x+\int_{\Omega} f_{1}\left(v_{m}\right) \phi d x
$$

for all $\phi \in W_{m}$ and

$$
\int_{\Omega} a_{2}\left(\left|\nabla v_{m}\right|^{p_{2}}\right)\left|\nabla v_{m}\right|^{p_{2}-2} \nabla v_{m} \nabla \varphi d x=\lambda_{2} \int_{\Omega} \frac{\varphi}{\left(v_{m}+\varepsilon\right)^{\beta_{2}}} d x+\int_{\Omega} f_{2}\left(u_{m}\right) \varphi d x
$$

for all $\varphi \in W_{m}$, which implies that $\left(u_{m}, v_{m}\right)$ is an approximate weak solution of problem (5.1).

Since $r$ does not depend on $m$ and $W_{m} \subset W_{0}^{1, N}(\Omega)$, for all $N$, then $\left(u_{m}\right)$ and $\left(v_{m}\right)$ are bounded sequences in $W_{0}^{1, N}(\Omega)$. Thus, for some subsequence, there exist $u, v \in W_{0}^{1, N}(\Omega)$ such that

$$
\begin{cases}u_{m} \rightarrow u & \text { in } W_{0}^{1, N}(\Omega), \\ u_{m} \rightarrow u & \text { in } L^{\theta}(\Omega), \theta \geq 1 \\ u_{m}(x) \rightarrow u(x) & \text { a.e. in } \Omega \\ \left|u_{m}(x)\right| \leq g_{1}(x) \in L^{\theta}(\Omega) & \text { a.e. in } \Omega, \theta \geq 1\end{cases}
$$

and

$$
\begin{cases}v_{m} \rightarrow v & \text { in } W_{0}^{1, N}(\Omega), \\ v_{m} \rightarrow v & \text { in } L^{\theta}(\Omega), \theta \geq 1 \\ v_{m}(x) \rightarrow v(x) & \text { a.e. in } \Omega \\ \left|v_{m}(x)\right| \leq g_{2}(x) \in L^{\theta}(\Omega) & \text { a.e. in } \Omega, \theta \geq 1\end{cases}
$$

Fix $k \in \mathbf{N}$ and consider $m \geq k$, then $W_{k} \subset W_{m}$ and

$$
\begin{aligned}
& \int_{\Omega} a_{1}\left(\left|\nabla u_{m}\right|^{p_{1}}\right)\left|\nabla u_{m}\right|^{p_{1}-2} \nabla u_{m} \nabla \phi_{k} d x \\
& =\lambda_{1} \int_{\Omega} \frac{\phi_{k}}{\left(u_{m}+\varepsilon\right)^{\beta_{1}}} d x+\int_{\Omega} f_{1}\left(v_{m}\right) \phi_{k} d x, \forall \phi_{k} \in W_{k}
\end{aligned}
$$


and

$$
\begin{aligned}
& \int_{\Omega} a_{2}\left(\left|\nabla v_{m}\right|^{p_{2}}\right)\left|\nabla v_{m}\right|^{p_{2}-2} \nabla v_{m} \nabla \varphi_{k} d x \\
& =\lambda_{2} \int_{\Omega} \frac{\varphi_{k}}{\left(v_{m}+\varepsilon\right)^{\beta_{2}}}+\int_{\Omega} f_{2}\left(u_{m}\right) \varphi_{k} d x, \forall \varphi_{k} \in W_{k} .
\end{aligned}
$$

Since $\phi_{k}, \varphi_{k} \in W_{m}$, note that

$$
\left|\frac{\phi_{k}}{\left(u_{m}+\varepsilon\right)^{\beta_{1}}}\right| \leq \frac{\left|\phi_{k}\right|}{\varepsilon^{\beta_{1}}} \in L^{1}(\Omega)
$$

and

By (5.14) and (5.15) we have

$$
\left|\frac{\varphi_{k}}{\left(v_{m}+\varepsilon\right)^{\beta_{2}}}\right| \leq \frac{\left|\varphi_{k}\right|}{\varepsilon^{\beta_{2}}} \in L^{1}(\Omega) .
$$

$$
\frac{\phi_{k}}{\left(u_{m}(x)+\varepsilon\right)^{\beta_{1}}} \rightarrow \frac{\phi_{k}}{(u(x)+\varepsilon)^{\beta_{1}}} \quad \text { a.e. in } \Omega
$$

and

$$
\frac{\varphi_{k}}{\left(v_{m}(x)+\varepsilon\right)^{\beta_{2}}} \rightarrow \frac{\varphi_{k}}{(v(x)+\varepsilon)^{\beta_{2}}} \quad \text { a.e. in } \Omega .
$$

Therefore, we use [6, Theorem 4.2] to obtain that

$$
\int_{\Omega} \frac{\phi_{k}}{\left(u_{m}+\varepsilon\right)^{\beta_{1}}} d x \rightarrow \int_{\Omega} \frac{\phi_{k}}{(u+\varepsilon)^{\beta_{1}}} d x
$$

and

$$
\int_{\Omega} \frac{\varphi_{k}}{\left(v_{m}+\varepsilon\right)^{\beta_{2}}} d x \rightarrow \int_{\Omega} \frac{\varphi_{k}}{(v+\varepsilon)^{\beta_{2}}} d x .
$$

Now, since $f_{i}$ are continuous functions, by (5.14) and (5.15) again we have

$$
f_{1}\left(v_{m}(x)\right) \phi_{k} \rightarrow f_{1}(v(x)) \phi_{k} \text { a.e in } \Omega
$$

and

$$
f_{2}\left(u_{m}(x)\right) \varphi_{k} \rightarrow f_{2}(u(x)) \varphi_{k} \text { a.e in } \Omega
$$

Using (2.1) we get

$$
\left|f_{1}\left(v_{m}(x)\right) \phi_{k}\right| \leq \delta_{1}\left|v_{m}(x)\right|^{p_{1}-1}\left|\phi_{k}\right|+C_{\delta_{1}}\left|v_{m}(x)\right|^{q_{1}-1} \exp \left(\alpha_{1}\left|v_{m}(x)\right|^{\frac{N}{N-1}}\right)\left|\phi_{k}\right|
$$

and

$$
\left|f_{2}\left(u_{m}(x)\right) \varphi_{k}\right| \leq \delta_{2}\left|u_{m}(x)\right|^{p_{2}-1}\left|\varphi_{k}\right|+C_{\delta_{2}}\left|u_{m}(x)\right|^{q_{2}-1} \exp \left(\alpha_{2}\left|u_{m}(x)\right|^{\frac{N}{N-1}}\right)\left|\varphi_{k}\right| .
$$

We will need to prove that the functions $\widehat{g_{1}}, \widehat{g_{2}}: \mathbf{R} \rightarrow \mathbf{R}$ defined by

$$
\widehat{g_{1}}\left(v_{m}(x)\right):=\delta_{1}\left|v_{m}(x)\right|^{p_{1}-1}\left|\phi_{k}\right|+C_{\delta_{1}}\left|v_{m}(x)\right|^{q_{1}-1} \exp \left(\alpha_{1}\left|v_{m}(x)\right|^{\frac{N}{N-1}}\right)\left|\phi_{k}\right|
$$

and

$$
\widehat{g_{2}}\left(u_{m}(x)\right):=\delta_{2}\left|u_{m}(x)\right|^{p_{2}-1}\left|\varphi_{k}\right|+C_{\delta_{2}}\left|u_{m}(x)\right|^{q_{2}-1} \exp \left(\alpha_{2}\left|u_{m}(x)\right|^{\frac{N}{N-1}}\right)\left|\varphi_{k}\right|
$$

satisfy

$$
\left|f_{1}\left(v_{m}(x)\right) \phi_{k}\right| \leq \widehat{g_{1}}\left(v_{m}(x)\right) \in L^{1}(\Omega)
$$

and

$$
\left|f_{2}\left(u_{m}(x)\right) \varphi_{k}\right| \leq \widehat{g_{2}}\left(u_{m}(x)\right) \in L^{1}(\Omega) .
$$


It is sufficiently to show that $\widehat{g_{1}}\left(v_{m}(x)\right)$ and $\widehat{g_{2}}\left(u_{m}(x)\right)$ are convergent in $L^{1}(\Omega)$. We will prove only the first inequality, because the second follows of the same reasoning. Indeed, since $2 \leq p_{1}<N$ we invoke (5.15) to get

$$
\left|v_{m}(x)\right|^{p_{1}-1}\left|\phi_{k}\right| \rightarrow|v(x)|^{p_{1}-1}\left|\phi_{k}\right| \quad \text { a.e. in } \Omega
$$

and

$$
\left|v_{m}(x)\right|^{p_{1}-1}\left|\phi_{k}\right| \leq g_{2}(x)^{p_{1}-1}\left|\phi_{k}\right| \in L^{1}(\Omega) .
$$

It follows from (5.24), (5.25) and [1, Theorem 4.2] that

$$
\int_{\Omega}\left|v_{m}\right|^{p_{1}-1}\left|\phi_{k}\right| d x \rightarrow \int_{\Omega}|v|^{p_{1}-1}\left|\phi_{k}\right| d x
$$

Furthermore, from (5.15) again we get

$$
\left|v_{m}(x)\right|^{q_{1}-1} \exp \left(\alpha_{1}\left|v_{m}(x)\right|^{\frac{N}{N-1}}\right) \rightarrow|v(x)|^{q_{1}-1} \exp \left(\alpha_{1}|v(x)|^{\frac{N}{N-1}}\right) \quad \text { a.e. in } \Omega \text {. }
$$

Now, considering $s, s^{\prime}>1$ such that $\frac{1}{s}+\frac{1}{s^{\prime}}=1$, we use (5.15) and the fact that $q_{1}>N$ to obtain

$$
\left|v_{m}\right|^{q_{1}-1} \rightarrow|v|^{q_{1}-1} \quad \text { in } L^{s^{\prime}}(\Omega) .
$$

Moreover, by (5.11) we have

$$
\begin{aligned}
\int_{\Omega} \exp \left(\alpha_{1} s\left|v_{m}(x)\right|^{\frac{N}{N-1}}\right) d x & =\int_{\Omega} \exp \left(\alpha_{1} s\left\|v_{m}(x)\right\|_{1, N}^{\frac{N}{N-1}}\left(\frac{\left|v_{m}(x)\right|}{\left\|v_{m}(x)\right\|_{1, N}}\right)^{\frac{N}{N-1}}\right) d x \\
& \leq \int_{\Omega} \exp \left(\alpha_{1} s r^{\frac{N}{N-1}}\left(\frac{\left|v_{m}(x)\right|}{\left\|v_{m}(x)\right\|_{1, N}}\right)^{\frac{N}{N-1}}\right) d x
\end{aligned}
$$

and applying the Theorem 2.1 we obtain

$$
\int_{\Omega} \exp \left(\alpha_{1} s\left|v_{m}(x)\right|^{\frac{N}{N-1}}\right) d x \leq \int_{\Omega} \exp \left(\alpha_{N}\left(\frac{\left|v_{m}(x)\right|}{\left\|v_{m}(x)\right\|_{1, N}}\right)^{\frac{N}{N-1}}\right) d x \leq M_{1} .
$$

Hence, by (5.28), (5.29) and Hölder's inequality we get

$$
\begin{aligned}
\int_{\Omega}\left|v_{m}\right|^{q_{1}-1} \exp \left(\alpha_{1}\left|v_{m}\right|^{\frac{N}{N-1}}\right) d x & \leq\left(\int_{\Omega}\left|u_{m}\right|^{\left(q_{1}-1\right) s^{\prime}} d x\right)^{\frac{1}{s^{\prime}}}\left(\int_{\Omega} \exp \left(\alpha_{1} s\left|v_{m}\right|^{\frac{N}{N-1}}\right) d x\right)^{\frac{1}{s}} \\
& \leq\left|v_{m}\right|_{L^{s^{\prime}}(\Omega)}^{q_{1}-1} M_{1}^{\frac{1}{s}}=\overline{M_{1}} .
\end{aligned}
$$

We use (5.27), (5.30) and [26, Theorem 4.8] to conclude that

$$
\left|v_{m}\right|^{q_{1}-1} \exp \left(\alpha_{1}\left|v_{m}\right|^{\frac{N}{N-1}}\right) \rightarrow|v|^{q_{1}-1} \exp \left(\alpha_{1}|v|^{\frac{N}{N-1}}\right) .
$$

It follows from (5.31) that

$$
\int_{\Omega}\left|v_{m}\right|^{q_{1}-1} \exp \left(\alpha_{1}\left|v_{m}\right|^{\frac{N}{N-1}}\right)\left|\phi_{k}\right| d x \rightarrow \int_{\Omega}\left|v^{q_{1}-1} \exp \left(\alpha_{1}|v|^{\frac{N}{N-1}}\right)\right| \phi_{k} \mid d x .
$$

Therefore, by (5.26) and (5.32) we prove that

$$
\int_{\Omega} \widehat{g_{1}}\left(v_{m}(x)\right) d x \rightarrow \delta_{1} \int_{\Omega}|v(x)|^{p_{1}-1}\left|\phi_{k}\right| d x+C_{\delta_{1}} \int_{\Omega}|v(x)|^{q_{1}-1} \exp \left(\alpha_{1}|v(x)|^{\frac{N}{N-1}}\right)\left|\phi_{k}\right| d x,
$$

which shows the identity (5.22). 
Then, we use $(5.20),(5.21),(5.22),(5.23)$ and $[6$, Theorem 4.2$]$ to conclude that

$$
\int_{\Omega} f_{1}\left(v_{m}\right) \phi_{k} d x \rightarrow \int_{\Omega} f_{1}(v) \phi_{k} d x
$$

and

$$
\int_{\Omega} f_{2}\left(u_{m}\right) \varphi_{k} d x \rightarrow \int_{\Omega} f_{2}(u) \varphi_{k} d x
$$

The next step is to show that

$$
\int_{\Omega} a_{1}\left(\left|\nabla u_{m}\right|^{p_{1}}\right)\left|\nabla u_{m}\right|^{p_{1}-2} \nabla u_{m} \nabla \phi_{k} d x \rightarrow \int_{\Omega} a_{1}\left(|\nabla u|^{p_{1}}\right)|\nabla u|^{p_{1}-2} \nabla u \nabla \phi_{k} d x
$$

and

$$
\int_{\Omega} a_{2}\left(\left|\nabla v_{m}\right|^{p_{2}}\right)\left|\nabla v_{m}\right|^{p_{2}-2} \nabla v_{m} \nabla \varphi_{k} d x \rightarrow \int_{\Omega} a_{2}\left(|\nabla v|^{p_{2}}\right)|\nabla v|^{p_{2}-2} \nabla u \nabla \varphi_{k} d x
$$

To this end, we will use $\left(a_{2}\right)$ and the same reasoning in [18, Lemma 2.4] to obtain $0 \leq C\left\|u_{m}-u\right\|_{1, N}$

$$
\begin{aligned}
& \leq \int_{\Omega} a_{1}\left(\left|\nabla u_{m}\right|^{p_{1}}\right)\left|\nabla u_{m}\right|^{p_{1}} d x-\int_{\Omega} a_{1}\left(\left|\nabla u_{m}\right|^{p_{1}}\right)\left|\nabla u_{m}\right|^{p_{1}-2} \nabla u_{m} \nabla u d x+o_{n}(1) \\
& =\lambda_{1} \int_{\Omega} \frac{u_{m}}{\left(u_{m}+\varepsilon\right)^{\beta_{1}}} d x+\int_{\Omega} f_{1}\left(v_{m}\right) u_{m} d x-\lambda_{1} \int_{\Omega} \frac{u}{\left(u_{m}+\varepsilon\right)^{\beta_{1}}} d x-\int_{\Omega} f_{1}\left(v_{m}\right) u=o_{n}(1),
\end{aligned}
$$

where

$$
o_{n}(1)=\int_{\Omega} a_{1}\left(|\nabla u|^{p_{1}}\right)|\nabla u|^{p_{1}} d x-\int_{\Omega} a_{1}\left(|\nabla u|^{p_{1}}\right)|\nabla u|^{p_{1}-2} \nabla u_{m} \nabla u d x
$$

and

$$
\begin{aligned}
0 & \leq C\left\|v_{m}-v\right\|_{1, N} \\
& \leq \int_{\Omega} a_{2}\left(\left|\nabla v_{m}\right|^{p_{2}}\right)\left|\nabla v_{m}\right|^{p_{2}} d x-\int_{\Omega} a_{2}\left(\left|\nabla v_{m}\right|^{p_{2}}\right)\left|\nabla v_{m}\right|^{p_{2}-2} \nabla v_{m} \nabla v d x+o_{n}(1) \\
& =\lambda_{2} \int_{\Omega} \frac{v_{m}}{\left(v_{m}+\varepsilon\right)^{\beta_{2}}} d x+\int_{\Omega} f_{2}\left(u_{m}\right) v_{m} d x-\lambda_{2} \int_{\Omega} \frac{v}{\left(v_{m}+\varepsilon\right)^{\beta_{2}}} d x-\int_{\Omega} f_{2}\left(u_{m}\right) v d x=o_{n}(1),
\end{aligned}
$$

where

$$
o_{n}(1)=\int_{\Omega} a_{2}\left(|\nabla v|^{p_{2}}\right)|\nabla v|^{p_{2}} d x-\int_{\Omega} a_{2}\left(|\nabla v|^{p_{2}}\right)|\nabla v|^{p_{2}-2} \nabla v_{m} \nabla v d x .
$$

Hence,

$$
\left\|u_{m}-u\right\|_{1, N}=o_{n}(1) \text { and }\left\|v_{m}-v\right\|_{1, N}=o_{n}(1),
$$

which implies that

$$
u_{m} \rightarrow u \text { in } W_{0}^{1, N}(\Omega)
$$

and

$$
v_{m} \rightarrow v \text { in } W_{0}^{1, N}(\Omega)
$$

Now, we know that the functions defined by

$$
E_{1}(u)=\int_{\Omega} a_{1}\left(|\nabla u|^{p_{1}}\right)|\nabla u|^{p_{1}-2} \nabla u \nabla \phi_{k} d x
$$

and

$$
E_{2}(v)=\int_{\Omega} a_{2}\left(|\nabla v|^{p_{2}}\right)|\nabla v|^{p_{2}-2} \nabla v \nabla \varphi_{k} d x
$$


are continuous. Then, by (5.37) and (5.38) we get the convergences (5.35) and (5.36).

Letting $m \rightarrow \infty$ in (5.16) and (5.17), we use (5.18), (5.19), (5.33), (5.34), (5.35) and (5.36) to conclude that

$$
\int_{\Omega} a_{1}\left(|\nabla u|^{p_{1}}\right)|\nabla u|^{p_{1}-2} \nabla u \nabla \phi_{k} d x=\lambda_{1} \int_{\Omega} \frac{\phi_{k}}{(u+\varepsilon)^{\beta_{1}}} d x+\int_{\Omega} f_{1}(v) \phi_{k} d x,
$$

and

$$
\int_{\Omega} a_{2}\left(|\nabla v|^{p_{2}}\right)|\nabla v|^{p_{2}-2} \nabla v \nabla \varphi_{k} d x=\lambda_{2} \int_{\Omega} \frac{\varphi_{k}}{(v+\varepsilon)^{\beta_{2}}} d x+\int_{\Omega} f_{2}(u) \varphi_{k} d x
$$

for all $\phi_{k}, \varphi_{k} \in W_{k}$.

Since $\left[W_{k}\right]_{k \in \mathbf{N}}$ is dense in $W_{0}^{1, N}(\Omega)$, we have

$$
\phi_{k} \rightarrow \phi \quad \text { as } k \rightarrow \infty
$$

and

$$
\varphi_{k} \rightarrow \varphi \quad \text { as } k \rightarrow \infty
$$

Then,

$$
\begin{aligned}
\int_{\Omega} a_{1}\left(|\nabla u|^{p_{1}}\right)|\nabla u|^{p_{1}-2} \nabla u \nabla \phi_{k} d x & \rightarrow \int_{\Omega} a_{1}\left(|\nabla u|^{p_{1}}\right)|\nabla u|^{p_{1}-2} \nabla u \nabla \phi d x \\
\int_{\Omega} a_{2}\left(|\nabla v|^{p_{2}}\right)|\nabla v|^{p_{2}-2} \nabla v \nabla \varphi_{k} d x & \rightarrow \int_{\Omega} a_{2}\left(|\nabla v|^{p_{2}}\right)|\nabla v|^{p_{2}-2} \nabla v \nabla \varphi d x \\
\int_{\Omega} \frac{\phi_{k}}{(u+\varepsilon)^{\beta_{1}}} d x & \rightarrow \int_{\Omega} \frac{\phi}{(u+\varepsilon)^{\beta_{1}}} d x \\
\int_{\Omega} \frac{\varphi_{k}}{(v+\varepsilon)^{\beta_{2}}} d x & \rightarrow \int_{\Omega} \frac{\varphi}{(v+\varepsilon)^{\beta_{2}}} d x \\
\int_{\Omega} f_{1}(v) \phi_{k} d x & \rightarrow \int_{\Omega} f_{1}(v) \phi d x
\end{aligned}
$$

and

$$
\int_{\Omega} f_{2}(u) \varphi_{k} d x \rightarrow \int_{\Omega} f_{2}(u) \varphi d x
$$

Therefore, since $\phi, \varphi \in W_{0}^{1, N}(\Omega)$ are arbitrary, it follows from (5.39) - (5.46) that

$$
\int_{\Omega} a_{1}\left(|\nabla u|^{p_{1}}\right)|\nabla u|^{p_{1}-2} \nabla u \nabla \phi d x=\lambda_{1} \int_{\Omega} \frac{\phi}{(u+\varepsilon)^{\beta_{1}}} d x+\int_{\Omega} f_{1}(v) \phi d x
$$

and

$$
\int_{\Omega} a_{2}\left(|\nabla v|^{p_{2}}\right)|\nabla v|^{p_{2}-2} \nabla v \nabla \varphi d x=\lambda_{2} \int_{\Omega} \frac{\varphi}{(v+\varepsilon)^{\beta_{2}}} d x+\int_{\Omega} f_{2}(u) \varphi d x
$$

for all $\phi, \varphi \in W_{0}^{1, N}(\Omega)$, which shows that $(u, v)$ is a weak solution of the problem (5.1). Furthermore, arguing as in the scalar case we conclude that $u, v>0$ in $\Omega$. 


\section{Proof of the Theorem 1.2}

For each $n \in \mathbf{N}$, let $\varepsilon=\frac{1}{n}, u_{\frac{1}{n}}=u_{n}$ and $v_{\frac{1}{n}}=v_{n}$ be, where $\left(u_{n}, v_{n}\right)$ is a solution of auxiliary problem (5.1)

$$
\begin{cases}-\operatorname{div}\left(a_{1}\left(\left|\nabla u_{n}\right|^{p_{1}}\right)\left|\nabla u_{n}\right|^{p_{1}-2} \nabla u_{n}\right)=\frac{\lambda_{1}}{\left(u_{n}+\frac{1}{n}\right)^{\beta_{1}}}+f_{1}\left(v_{n}\right) & \text { in } \Omega, \\ -\operatorname{div}\left(a_{2}\left(\left|\nabla v_{n}\right|^{p_{2}}\right)\left|\nabla v_{n}\right|^{p_{2}-2} \nabla v_{n}\right)=\frac{\lambda_{2}}{\left(v_{n}+\frac{1}{n}\right)^{\beta_{2}}}+f_{2}\left(u_{n}\right) & \text { in } \Omega, \\ u_{n}, v_{n}>0 & \text { in } \Omega, \\ u_{n}, v_{n}=0 & \text { on } \partial \Omega,\end{cases}
$$

obtained by the Lemma 5.1. Note that, from $\left(f_{3}\right)$ we obtain

$$
-\operatorname{div}\left(a_{1}\left(\left|\nabla u_{n}\right|^{p_{1}}\right)\left|\nabla u_{n}\right|^{p_{1}-2} \nabla u_{n}\right) \geq \frac{\lambda_{1}}{\left(u_{n}+v_{n}+1\right)^{\beta_{1}}}+\left|v_{n}\right|^{\gamma_{1}-1} \text { in } \Omega
$$

and as the function $t \mapsto \frac{\lambda_{1}}{\left(u_{n}+t+1\right)^{\beta_{1}}}+t^{\gamma_{1}-1}$, for all $t \geq 0$, attains a positive minimum $z_{1}$. Then,

$$
-\operatorname{div}\left(a_{1}\left(\left|\nabla u_{n}\right|^{p_{1}}\right)\left|\nabla u_{n}\right|^{p_{1}-2} \nabla u_{n}\right) \geq z_{1}>0 \text { in } \Omega .
$$

By virtue from Minty-Browder's Theorem [6, Theorem 5.15], we use the unique positive solution of the problem

$$
\begin{cases}-\operatorname{div}\left(a_{1}\left(\left|\nabla w_{1}\right|^{p_{1}}\right)\left|\nabla w_{1}\right|^{p_{1}-2} \nabla w_{1}\right)=z_{1} & \text { in } \Omega \\ w_{1}>0 & \text { in } \Omega \\ w_{1}=0 & \text { on } \partial \Omega\end{cases}
$$

to get that the solution $w_{1}$ bound from below the solution $u_{n}$. Therefore,

$$
\begin{cases}-\operatorname{div}\left(a_{1}\left(\left|\nabla u_{n}\right|^{p_{1}}\right)\left|\nabla u_{n}\right|^{p_{1}-2} \nabla u_{n}\right) \geq-\operatorname{div}\left(a_{1}\left(\left|\nabla w_{1}\right|^{p_{1}}\right)\left|\nabla w_{1}\right|^{p_{1}-2} \nabla w_{1}\right) & \text { in } \Omega, \\ u_{n}=w_{1} & \text { on } \partial \Omega .\end{cases}
$$

Hence, we use the Lemma 2.1 to conclude that

$$
u_{n}(x) \geq w_{1}(x)>0 \text { in } \Omega, \forall n \in \mathbf{N} .
$$

Similarly, we prove that

$$
v_{n}(x) \geq w_{2}(x)>0 \text { in } \Omega \forall n \in \mathbf{N},
$$

where $w_{2}$ satisfies

$$
\begin{cases}-\operatorname{div}\left(a_{2}\left(\left|\nabla w_{2}\right|^{p_{2}}\right)\left|\nabla w_{2}\right|^{p_{2}-2} \nabla w_{2}\right)=z_{2} & \text { in } \Omega \\ w_{2}>0 & \text { in } \Omega \\ w_{2}=0 & \text { in } \partial \Omega\end{cases}
$$

and $z_{2}$ is positive minimum of the function $t \mapsto \frac{\lambda_{2}}{\left(v_{n}+t+1\right)^{\beta_{2}}}+t^{\gamma_{2}-1}$.

Now, from (5.14) and (5.15) we get

$$
u_{m} \rightarrow u_{n} \text { in } W_{0}^{1, N}(\Omega) \text { as } m \rightarrow+\infty
$$

and

$$
v_{m} \rightarrow v_{n} \text { in } W_{0}^{1, N}(\Omega) \text { as } m \rightarrow+\infty .
$$

It follows from (5.11) that

$$
\left\|u_{n}\right\|_{1, N} \leq \liminf _{m \rightarrow+\infty}\left\|u_{m}\right\|_{1, N} \leq \liminf _{m \rightarrow+\infty}\left\|\left(u_{m}, v_{m}\right)\right\| \leq r<1, \text { for all } n \in \mathbf{N}
$$


and

$$
\left\|v_{n}\right\|_{1, N} \leq \liminf _{m \rightarrow+\infty}\left\|v_{m}\right\|_{1, N} \leq \liminf _{m \rightarrow+\infty}\left\|\left(u_{m}, v_{m}\right)\right\| \leq r<1, \text { for all } n \in \mathbf{N} .
$$

Therefore, $r$ does not depend on $n$, which shows that $\left(u_{n}\right)$ and $\left(v_{n}\right)$ are a bounded sequences. Thus, since $W_{0}^{1, N}(\Omega)$ is a reflective Banach space, for some subsequence, there exist $u, v \in W_{0}^{1, N}(\Omega)$ such that

$$
\begin{cases}u_{n} \rightarrow u & \text { in } W_{0}^{1, N}(\Omega), \\ u_{n} \rightarrow u & \text { in } L^{\theta}(\Omega), \theta \geq 1, \\ u_{n}(x) \rightarrow u(x) & \text { a.e. in } \Omega, \\ \left|u_{n}(x)\right| \leq g_{1}(x) \in L^{\theta}(\Omega) & \text { a.e. in } \Omega, \theta \geq 1\end{cases}
$$

and

$$
\begin{cases}v_{n} \rightarrow v & \text { in } W_{0}^{1, N}(\Omega), \\ v_{n} \rightarrow v & \text { in } L^{\theta}(\Omega), \theta \geq 1 \\ v_{n}(x) \rightarrow v(x) & \text { a.e. in } \Omega \\ \left|v_{n}(x)\right| \leq g_{2}(x) \in L^{\theta}(\Omega) & \text { a.e. in } \Omega, \theta \geq 1\end{cases}
$$

Recall from (5.47) and (5.48) that

$$
\int_{\Omega} a_{1}\left(\left|\nabla u_{n}\right|^{p_{1}}\right)\left|\nabla u_{n}\right|^{p_{1}-2} \nabla u_{n} \nabla \phi d x=\lambda_{1} \int_{\Omega} \frac{\phi}{\left(u_{n}+\frac{1}{n}\right)^{\beta_{1}}} d x+\int_{\Omega} f_{1}\left(v_{n}\right) \phi
$$

for all $\phi \in W_{0}^{1, N}(\Omega) d x$ and

$$
\int_{\Omega} a_{2}\left(\left|\nabla v_{n}\right|^{p_{2}}\right)\left|\nabla v_{n}\right|^{p_{2}-2} \nabla v_{n} \nabla \varphi d x=\lambda_{2} \int_{\Omega} \frac{\varphi}{\left(v_{n}+\frac{1}{n}\right)^{\beta_{2}}} d x+\int_{\Omega} f_{2}\left(u_{n}\right) \varphi d x
$$

for all $\varphi \in W_{0}^{1, N}(\Omega)$.

Since $f_{i}$ are continuous functions, by (6.5) and (6.6), we have

$$
f_{1}\left(v_{n}(x)\right) \phi \rightarrow f_{1}(v(x)) \phi \text { a.e. in } \Omega
$$

and

$$
f_{2}\left(u_{n}(x)\right) \varphi \rightarrow f_{2}(u(x)) \varphi \text { a.e. in } \Omega .
$$

By same computation in (5.22) and (5.23), we obtain

$$
\left|f_{1}\left(v_{n}(x)\right) \phi\right| \leq \widehat{g_{1}}\left(v_{n}(x)\right)
$$

and

$$
\left|f_{2}\left(u_{n}(x)\right) \varphi\right| \leq \widehat{g_{2}}\left(u_{n}(x)\right)
$$

such that $\widehat{g}_{i}$ converge in $\in L^{1}(\Omega)$. Then, we use [6, Theorem 4.2] to conclude that

$$
\int_{\Omega} f_{1}\left(v_{n}\right) \phi d x \rightarrow \int_{\Omega} f_{1}(v) \phi d x, \forall \phi \in W_{0}^{1, N}(\Omega)
$$

and

$$
\int_{\Omega} f_{2}\left(u_{n}\right) \varphi d x \rightarrow \int_{\Omega} f_{2}(u) \varphi, \forall \varphi \in W_{0}^{1, N}(\Omega) d x .
$$

Now, by same computation in (5.35) and (5.36), we have

$$
\int_{\Omega} a_{1}\left(\left|\nabla u_{n}\right|^{p_{1}}\right)\left|\nabla u_{n}\right|^{p_{1}-2} \nabla u_{n} \nabla \phi d x \rightarrow \int_{\Omega} a_{1}\left(|\nabla u|^{p_{1}}\right)|\nabla u|^{p_{1}-2} \nabla u \nabla \phi d x
$$


for all $\phi \in W_{0}^{1, N}(\Omega)$ and

$$
\int_{\Omega} a_{2}\left(\left|\nabla v_{n}\right|^{p_{2}}\right)\left|\nabla v_{n}\right|^{p_{2}-2} \nabla v_{n} \nabla \varphi d x \rightarrow \int_{\Omega} a_{2}\left(|\nabla v|^{p_{2}}\right)|\nabla v|^{p_{2}-2} \nabla v \nabla \varphi d x,
$$

for all $\varphi \in W_{0}^{1, N}(\Omega)$.

Note that, from (6.5) and (6.6) again we get

$$
\frac{\phi}{\left(u_{n}(x)+\frac{1}{n}\right)^{\beta_{1}}} \rightarrow \frac{\phi}{u(x)^{\beta_{1}}} \text { a.e. in } \Omega
$$

and

$$
\frac{\varphi}{\left(v_{n}(x)+\frac{1}{n}\right)^{\beta_{2}}} \rightarrow \frac{\varphi}{v(x)^{\beta_{2}}} \text { a.e. in } \Omega .
$$

Moreover, by virtue from (6.1), (6.4) and $\left(a_{1}\right)$, we can argue as in [23] to obtain that $w_{1}, w_{2} \in C^{1}(\bar{\Omega})$. Consequently, we use (6.1), (6.4) again to conclude

$$
\frac{\partial w_{1}}{\partial \eta}, \frac{\partial w_{2}}{\partial \eta}<0 \quad \text { on } \partial \Omega
$$

Then, for each $x \in \Omega$, it follows from (6.2) and (6.3) that

$$
u_{n}(x) \geq w_{1}(x)>C d(x)>0 \quad \text { and } \quad v_{n}(x) \geq w_{2}(x)>C d(x)>0,
$$

where $d(x)=\operatorname{dist}(x, \partial \Omega)$ and $C$ is positive constant that does not depend on $x$. Thus,

$$
\int_{\Omega} \frac{\phi}{\left(u_{n}(x)+\frac{1}{n}\right)^{\beta_{1}}} d x \leq \int_{\Omega} \frac{\phi}{u_{n}(x)^{\beta_{1}}} d x \leq \int_{\Omega} \frac{\phi}{C d(x)^{\beta_{1}}} d x
$$

and

$$
\int_{\Omega} \frac{\varphi}{\left(v_{n}(x)+\frac{1}{n}\right)^{\beta_{2}}} d x \leq \int_{\Omega} \frac{\varphi}{v_{n}(x)^{\beta_{2}}} d x \leq \int_{\Omega} \frac{\varphi}{C d(x)^{\beta_{2}}} d x
$$

Hence, we invoke Theorem 2.2 to obtain $\left|\frac{\phi}{C d(x)^{\beta_{1}}}\right|,\left|\frac{\varphi}{C d(x)^{\beta_{2}}}\right| \in L^{r}(\Omega)$ and $C_{17}, C_{18}>$ 0 such that

$$
\int_{\Omega} \frac{\phi}{\left(u_{n}(x)+\frac{1}{n}\right)^{\beta_{1}}} d x \leq C_{17}\|\phi\|_{1, N}
$$

and

$$
\int_{\Omega} \frac{\varphi}{\left(v_{n}(x)+\frac{1}{n}\right)^{\beta_{2}}} d x \leq C_{18}\|\varphi\|_{1, N} .
$$

Therefore, by (6.13), (6.14), (6.15), (6.16) and [6, Theorem 4.2] we get

$$
\int_{\Omega} \frac{\phi}{\left(u_{n}+\frac{1}{n}\right)^{\beta_{1}}} d x \rightarrow \int_{\Omega} \frac{\phi}{u^{\beta_{1}}} d x, \forall \phi \in W_{0}^{1, N}(\Omega)
$$

and

$$
\int_{\Omega} \frac{\varphi}{\left(v_{n}+\frac{1}{n}\right)^{\beta_{2}}} d x \rightarrow \int_{\Omega} \frac{\varphi}{v^{\beta_{2}}} d x, \quad \forall \varphi \in W_{0}^{1, N}(\Omega) .
$$

Letting $n \rightarrow+\infty$ in (6.7) and (6.8), we use (6.9), (6.10), (6.11), (6.12), (6.17) and (6.18) to conclude that

$$
\int_{\Omega} a_{1}\left(|\nabla u|^{p_{1}}\right)|\nabla u|^{p_{1}-2} \nabla u \nabla \phi d x=\lambda_{1} \int_{\Omega} \frac{\phi}{u^{\beta_{1}}} d x+\int_{\Omega} f_{1}(v) \phi d x, \quad \forall \phi \in W_{0}^{1, N}(\Omega)
$$


and

$$
\int_{\Omega} a_{2}\left(|\nabla v|^{p_{2}}\right)|\nabla v|^{p_{2}-2} \nabla v \nabla \varphi d x=\lambda_{2} \int_{\Omega} \frac{\varphi}{v^{\beta_{2}}} d x+\int_{\Omega} f_{2}(u) \varphi d x, \quad \forall \varphi \in W_{0}^{1, N}(\Omega),
$$

which proves that $(u, v) \in W^{1, N}(\Omega) \times W^{1, N}(\Omega)$ is a weak solution of the problem $(1.2)$.

\section{References}

[1] Alves, C. O., F. J. S. A. CorrêA, and J. V. A. GonçAlves: Existence of solutions for some classes of singular Hamiltonian systems. - Adv. Nonlinear Stud. 5:2, 2005, 265-278.

[2] Aounoui, S.: On some quasilinear equation with critical exponential growth at infinity and a singular behavior at the origin. - J. Elliptic Parabol. Equ. 4:1, 2018, 27-50.

[3] Arora, R., J. Giacomoni, T. Mukherjee, and K. Sreenadh: Adams-Moser-Trudinger inequality in the Cartesian product of Sobolev spaces and its applications. - Rev. R. Acad. Cienc. Exactas Fís. Nat. Ser. A Mat. RACSAM 114:3, 2020, 111.

[4] Boccardo, L.: A Dirichlet problem with singular and supercritical nonlinearities. - Nonlinear Anal. 75:12, 2012, 4436-4440.

[5] Boccardo, L., and L. Orsina: Semilinear elliptic equations with singular nonlinearities. Calc. Var. Partial Differential Equations 37:3-4, 2010, 363-380.

[6] Brezis, H.: Analyse fonctinelle. - Masson, Paris, 1983.

[7] Callegari, A., and A. Nachman: A nonlinear singular boundary value problem in the theory of pseudoplastic fluids. - SIAM J. Appl. Math. 38:2, 1980, 275-281.

[8] Callegari A., and A. Nachman: Some singular nonlinear differential equations arising in boundary layer theory. - J. Math. Anal. Appl. 64:1, 1978, 96-105.

[9] Canino, A., and M. Degiovanni: A variational approach to a class of singular semilinear elliptic equations. - J. Convex Anal. 11:1, 2004, 147-162.

[10] Canino, A., M. Grandinetti, and B. Sciunzi: A jumping problem for some singular semilinear elliptic equations. - Adv. Nonlinear Stud. 14:4, 2014, 1037-1054.

[11] Cherfils, L., and Y. IL'YAsov: On the stationary solutions of generalized reaction difusion equations with $p \& q$-Laplacian. - Commun. Pure Appl. Anal. 4:1, 2005, 9-22.

[12] Corrêa, A. S., F. J. S. A. CorrêA, and G. M. Figueiredo: Existence of positive solution for a singular system involving general quasilinear operators. - Differ. Equ. Appl. 6:4, 2014, 481-494.

[13] Corrêa, A. S., F. J. S. A. CorrêA, and G. M. Figueiredo: Positive solution for a class of $p \& q$ singular elliptic equation. - Nonlinear Anal. Real World Appl. 16, 2014, 163-169.

[14] Crandall, M. G., P. H. Rabinowitz, and L. Tatar: On a Dirichlet problem with a singular nonlinearity. - Commun. Partial Differential Equations 2:2, 1997, 193-222.

[15] Dávila, J., and M. Montenegro: Positive versus free boundary solutions to a singular elliptic equation. - J. Anal. Math. 90, 2003, 303-335.

[16] Dhanya, R., S. Prashanth, K. Sreenadh, and S. Tiwari: Critical growth elliptic problem in $\mathbf{R}^{2}$ with singular discontinuous nonlinearities. - Adv. Differential Equations 19:5-6, 2014, 409-440.

[17] Felmer, P., A. QuaAs, and B. Sirakov: Existence and regularity results for fully nonlinear equations with singularities. - Math. Ann. 354:1, 2012, 377-400.

[18] Figueiredo, G. M.: Existence of positive solutions for a class of $p \& q$ elliptic problem with critical growth on $\mathbf{R}^{N}$. - J. Math. Anal. Appl. 378:2, 2011, 507-518.

[19] Ghergu, M., and V. RÃdulescu: On a class of sublinear singular elliptic problems with convection term. - J. Math. Anal. Appl. 311:2, 2005, 635-646. 
[20] Giacomoni, J., S. Prashanth, and K. Sreenadh: Uniqueness and multiplicity results for $N$-Laplace equation with critical and singular nonlinearity in a ball. - Asymptot. Anal. 61:3-4, 2009, 195-227.

[21] Gilbarg, D., and N. Trudinger: Elliptic partial differential equations of second order. 2nd edition. - Springer, 1983.

[22] HAI, D. D.: On an asymptotically linear singular boundary value problems. - Topol. Methods Nonlinear Anal. 39:1, 2012, 83-92.

[23] He, C., and G. LI: The regularity of weak solutions to nonlinear scalar field elliptic equations containing $p \& q$-Laplacians. - Ann. Acad. Sci. Fenn. Math. 33:2, 2008, 337-371.

[24] Hernández, J., F. J. Mancebo, and J. M. Vega: Positive solutions for singular semilinear elliptic systems. - Adv. Differential Equations 13:9-10, 2008, 857-880.

[25] Kavian, O.: Inegalitè de Hardy-Sobolev et applications. - Tèse de Doctorate de 3 eme cycle, Universitè de Paris VI, 1978.

[26] Kavian, O.: Introdution à la théorie des points critiques et applications aux problèms elliptiques. - Springer, Heidelberg, 1983.

[27] Kesavan, S.: Topics in functional analysis and applications. - Wiley, New Jersey, 1989.

[28] Lazer, A. C., and P. J. MCKennA: On a singular nonlinear elliptic boundary-value problem. - Proc. Amer. Math. Soc. 111:3, 1991, 721-730.

[29] Lieb, E. H., and M. Loss: Analysis. - Grad. Stud. Math. 14, Amer. Math. Soc., 1997.

[30] Montenegro, M., and A. SuÁrez: Existence of a positive solution for a singular system. Proc. Roy. Soc. Edinburgh Sect. A 140:2, 2010, 435-447.

[31] Moser, J.: A sharp form of an inequality by N. Trudinger. - Indiana Univ. Math. J. 20, 1970/71, 1077-1092.

[32] Perera, K., and E. A. B. Silva: Multiple positive solutions of singular elliptic problems. Differential Integral Equations 23:5-6, 2010, 435-444.

[33] Prashanth, S., S. Tiwhi, and K. Sreenadh: Very singular problems with critical nonlinearities in two dimensions. - Commun. Contemp. Math. 20:2, 2018, 1650067, 1-25.

[34] SAkAguchi, S.: Concavity properties of solutions to some degenerate quasilinear elliptic Dirichlet problems. - Ann. Scuola Norm. Sup. Pisa Cl. Sci. (4) 14:3, 1987, 403-421.

[35] Saoudi, K., and M. Kratou: Existence of multiple solutions for a singular and quasilinear equation. - Complex Var. Elliptic Equ. 60:7, 2015, 893-925.

[36] SAOudi, K.: Multiplicity results for a class of singular elliptic equation involving sublinear Neumann boundary condition in $\mathbf{R}^{2}$. - J. Fixed Point Theory Appl. 19:4, 2017, 2963-2984.

[37] ShI, J., and M. YAO: Positive solutions for elliptic equations with singular nonlinearity. Electron. J. Differential Equations 2005:4, 2005, 1-11.

[38] Strauss, W. A.: On weak solutions of semi-linear hyperbolic equations. - An. Acad. Brasil. Ci. 42, 1970, 645-651.

[39] Stuart, C.A.: Existence and approximation of solutions of non-linear elliptic equations. Math. Z. 147:1, 1976, 53-63.

[40] Trudinger, N. S.: On imbeddings into Orlicz spaces and applications. - J. Math. Mech. 17, 1967, 473-483.

[41] WU, M., and Z. YANG: A class of $p-q$-Laplacian type equation with potentials eigenvalue problem in $\mathbf{R}^{N}$. - Bound. Value Probl., 2009, Art. ID 185319, 1-19.

[42] Zhang, Z.: Positive solutions of Lane-Emden systems with negative exponents: existence, boundary behavior and uniqueness. - Nonlinear Anal. 74:16, 2011, 5544-5553.

[43] Zhang, Z., and J. Yu: On a singular nonlinear Dirichlet problem with a convection term. SIAM J. Math. Anal. 32:4, 2000, 916-927. 